\title{
Relationship Between Blood Manganese and Bone Mineral Density and Bone Mineral Content in Adults: A Population-Based Cross-Sectional Study
}

\author{
Chao Wang \\ Xiangya Hospital Central South University \\ Yong Zhu \\ Xiangya Hospital Central South University \\ Haitao Long \\ Xiangya Hospital Central South University \\ Mingning Ou \\ Xiangya Hospital Central South University \\ Shushan Zhao ( $\square$ zhaoshuiquan@126.com) \\ Xiangya Hospital Central South University
}

\section{Research Article}

Keywords: National Health and Nutrition Examination Survey (NHANES), Bone mineral density (BMD), Bone mineral content (BMC), Manganese (Mn), Osteoporosis, Bone loss, Fragility fracture

Posted Date: January 19th, 2022

DOI: https://doi.org/10.21203/rs.3.rs-1246983/v1

License: @ (i) This work is licensed under a Creative Commons Attribution 4.0 International License. Read Full License 


\section{Abstract}

It has been reported that bone is the primary organ for manganese $(\mathrm{Mn})$ accumulation, but the association between manganese and bone loss remains debatable. Therefore, this study aimed to evaluate the relationship between blood manganese and bone mineral density/bone mineral content (BMD/BMC) by using a representative sample from the National Health and Nutrition Examination Survey (NHANES). A total of 9732 subjects over the age of 18 with available data were enrolled in this study. The relationship between blood manganese and BMD/BMC of the total body, spine and femoral regions was evaluated using multivariate linear regression models. Subgroup analyses were also performed. We observed a negative association between blood manganese and BMD/BMC in the femoral neck and total body in the fully adjusted model, especially femoral neck BMD in women aged 50-70 years. In brief, people exposed to manganese should be aware of the increased risk of osteopenia or osteoporosis. Besides, due to the lack of available data, there are no definite values for the tolerable upper intake level (UL), average requirement (AR) and population reference intake (PRI) of manganese. The results of our study may provide some references for the establishment of AR, PRI and UL of Mn.

\section{Introduction}

Osteoporosis is a disease characterized by low bone mass and destruction of bone structure, resulting in decreased bone strength and increased risk of fractures $^{1}$. The incidence of osteoporosis will rise as the longevity of global population significantly raised, with significant increases in morbidity, disability and mortality, resulting in a significant socio-economic burden ${ }^{2}$. An estimated $16.2 \%$ of adults aged 65 and older in the United States had osteoporosis in the lumbar spine or femoral neck in $2010^{3}$. Oral bisphosphonates remain the most cost-effective first-line treatment. Since there are sufficient effective treatments for osteoporosis, better methods are needed to identify patients with high risk of fracture ${ }^{2}$.

Manganese $(\mathrm{Mn})$, the trace element, is critical for many physiological and biological processes, including body growth, enzymatic regulation reactions, immune function, metabolism, and bone growth ${ }^{4,5}$. However, excessive manganese in human body also causes harm to health. Manganese is partially absorbed through the gastrointestinal tract (3\%-5\%), vegetarians who eat foods rich in manganese, such as grains, legumes and nuts, and heavy tea drinkers, are likely having higher intake than the general population. People who smoke or inhale secondhand smoke are also exposed to higher levels of manganese than nonsmokers. Due to occupational exposure, the main source of entry for $\mathrm{Mn}$ is inhalation, which is also the primary source of clinically identified Mn intoxication ${ }^{6}$.

Bone is the primary organ for $\mathrm{Mn}$ accumulation ${ }^{7}$, but the association between $\mathrm{Mn}$ and bone loss remains debatable. Some previous studies have shown that women with osteoporosis have lower serum manganese levels than women with normal bone mineral density(BMD) ${ }^{8}$. While a previous case-control study suggested that retired female workers in the highest manganese exposure model (tertile 3 of $\mathrm{Mn}$-CEI) may be at higher risk of osteoporosis ${ }^{9}$. Besides, the European Food Safety Agency (EFSA) in 2013, judged that the available data to date are insufficient to establish an average requirement (Average Requirement, AR), as well as a reference intake for the population (Population Reference Intake, PRI) for Mn, and a tolerable upper intake level of manganese has not been established yet ${ }^{10}$.

However, among these epidemiological studies, few studies have examined the relationship between manganese status and bone mineral density $(\mathrm{BMD})$ /bone mineral content (BMC) in nationally representative samples. In this study, we used large datasets of total femur BMD/BMC, total spine BMD/BMC, and total body BMD/BMC released from 2013 to 2014 and 2017 to 2018 by the National Health and Nutrition Examination Survey (NHANES). We used blood manganese as a biomarker of manganese status. Therefore, the aim in this study was to evaluate the relationship between blood manganese and $\mathrm{BMD} / \mathrm{BMC}$ by using a representative sample from the National Health and Nutrition Examination Survey. A better understanding of the relationship between blood manganese and BMD may help to better identify patients with osteoporosis and prevent osteoporotic fractures.

\section{Methods}

\section{Study design and population}

The National Health and Nutrition Examination Survey (NHANES), a research program to assess the health and nutrition of adults and children in the United States, examining a nationally representative sample of approximately 5,000 people each year. In this study, we used NHANES data for 2013-2014 and 20172018. Of 19429 subjects, 12728 subjects with available blood manganese data were selected. After that, 2996 subjects lacking available Dual Energy X-ray Absorptiometry (DEXA) data were excluded. Finally, A total of 9732 subjects with available DEXA data on total body ( $n=3682)$, femur ( $n=3752)$, and spine $(n=2298)$ were enrolled. The Ethics Review Committee of the National Center for Health Statistics approved all NHANES protocols and obtained written informed consents from all participants ${ }^{11}$. The flow chart in Figure 1 also shows the selection process. All methods were performed in accordance with the relevant guidelines and regulations.

\section{Variables}

The exposure variable was blood manganese, which was measured using mass spectrometry after a simple dilution sample preparation step. The Hologic Discovery A is a fan beam X-ray bone densitometer used to perform DXA examinations and estimate bone mineral content (BMC) and bone mineral density (BMD). DXA total-body scans will be performed on all individuals aged 18 to 59 , while femur and spinal scans will be performed on samples aged 40 and older. Dependent variables were bone mineral density and bone mineral content of femur, lumbar spine and total body as measured by dual-energy $\mathrm{X}$-ray absorptiometry. The following categorical variables were included as covariates into our analysis: gender, race/ethnicity (Mexican American, other Hispanic, non-Hispanic White, non-Hispanic Black, other races), drinking, hypertension, hypercholesterolemia, diabetes, difficulty walking or climbing stairs, renal insufficiency, asthma, arthritis, congestive heart failure, stroke, coronary heart disease, angina, thyroid problems, COPD, smoking, family smokers and

Page $2 / 21$ 
osteoporosis. Our analysis included continuous covariates: age, body weight, standing height, and body mass index. Detailed information on blood manganese, BMD, BMC and covariates can be found on the NHANES website.

\section{Statistical Analysis}

Data were analyzed using R language, IBM SPSS Statistics version 26 (IBM, Armonk, New York, USA) and GraphPad Prism version 8.3.0 (GraphPad Software, San Diego, California USA). Means with standard deviation (SD) were used for continuous characteristic variables and categorical variables were expressed as percentages or frequencies. Differences in categorical variables between exposed groups were analyzed by Pearson's chi-square tests. One-way ANOVA was used to analyze the differences of continuous variables among groups. Blood manganese levels were classified according to quartiles (quartile 1 : < 25 th percentile, quartile 2: 25 th-50th percentile, quartile 3: 50th-75th percentile, quartile 4: > 75th percentile) and univariate and multivariable linear regression analysis were performed. In weighted univariate and multivariate linear regression models, we adjusted for potential confounders using an extended model approach to covariates to investigate the association between blood manganese levels and BMD/BMC (total femur, total spine, and total body). Including model 1 (unadjusted model), model 2 (adjusted for sex, age, race/ethnicity, and BMI), and model 3 (further adjusted for all relevant covariates in Table 1 ). The results of regression analysis were represented by $\beta$ regression coefficient, $95 \%$ confidence interval and $P$ value, $P<0.05$ (bilateral) was considered statistically significant. 
Table 1

Related demographic characteristics of participants Included in NHANES from 2013 to 2014 a

\begin{tabular}{|c|c|c|c|c|c|c|c|c|c|c|}
\hline \multirow[t]{2}{*}{ Characteristic } & & \multicolumn{6}{|l|}{ Femur } & \multicolumn{3}{|l|}{ Spine } \\
\hline & & Q1 & Q2 & Q3 & Q4 & total & $P$ value & Q1 & Q2 & Q3 \\
\hline \multirow[t]{2}{*}{ Gender } & male & 584 & 507 & 453 & 370 & 1914 & \multirow[t]{2}{*}{$<0.0001$} & 325 & 266 & 245 \\
\hline & female & 356 & 432 & 485 & 565 & 1838 & & 250 & 308 & 333 \\
\hline Age (years) & & $63.3 \pm 10.7$ & $62.7 \pm 10.4$ & $61.9 \pm 10.8$ & $60.6 \pm 10.7$ & $62.1 \pm 10.7$ & $<0.0001$ & $61.1 \pm 11.0$ & $59.8 \pm 10.6$ & $59.2 \pm 10.7$ \\
\hline \multirow[t]{5}{*}{ Race } & $\begin{array}{l}\text { Mexican } \\
\text { American }\end{array}$ & 77 & 115 & 123 & 140 & 455 & \multirow[t]{5}{*}{$<0.0001$} & 51 & 86 & 76 \\
\hline & $\begin{array}{l}\text { Other } \\
\text { Hispanic }\end{array}$ & 71 & 94 & 111 & 101 & 377 & & 46 & 65 & 70 \\
\hline & $\begin{array}{l}\text { Non- } \\
\text { Hispanic } \\
\text { White }\end{array}$ & 407 & 439 & 370 & 289 & 1505 & & 228 & 225 & 146 \\
\hline & $\begin{array}{l}\text { Non- } \\
\text { Hispanic } \\
\text { Black }\end{array}$ & 338 & 193 & 175 & 99 & 805 & & 220 & 129 & 103 \\
\hline & $\begin{array}{l}\text { Other } \\
\text { Race- } \\
\text { Including } \\
\text { Multi- } \\
\text { Racial }\end{array}$ & 47 & 98 & 159 & 306 & 610 & & 30 & 69 & 112 \\
\hline \multicolumn{2}{|l|}{ Weight(kg) } & $81.5 \pm 20.6$ & $81.4 \pm 17.8$ & $79.9 \pm 19.5$ & $76.5 \pm 18.5$ & $79.9 \pm 18.5$ & $<0.0001$ & $81.6 \pm 18.1$ & $81 \pm 20.1$ & $79.5 \pm 17.1$ \\
\hline \multicolumn{2}{|l|}{ Standing Height(cm) } & $168.7 \pm 10.2$ & $167 \pm 9.6$ & $165.4 \pm 10.0$ & $162.8 \pm 8.8$ & $166 \pm 8.8$ & $<0.0001$ & $168 \pm 9.9$ & $165.7 \pm 9.9$ & $164.8 \pm 8.7$ \\
\hline \multicolumn{2}{|l|}{ BMI (kg/m2) } & $28.6 \pm 6.5$ & $29.1 \pm 5.6$ & $29.1 \pm 6.0$ & $28.7 \pm 6.3$ & $28.9 \pm 6.3$ & 0.1123 & $28.8 \pm 6.0$ & $29.4 \pm 6.3$ & $29.2 \pm 6.4$ \\
\hline \multirow[t]{3}{*}{ Drinking alcohol } & YES & 176 & 155 & 133 & 90 & 554 & \multirow[t]{3}{*}{0.0001} & 100 & 82 & 76 \\
\hline & NO & 655 & 654 & 636 & 624 & 2569 & & 394 & 398 & 383 \\
\hline & N/A & 109 & 130 & 169 & 221 & 629 & & 81 & 94 & 119 \\
\hline \multirow[t]{3}{*}{ Hypertension } & YES & 493 & 495 & 477 & 427 & 1892 & \multirow[t]{3}{*}{0.0075} & 278 & 299 & 269 \\
\hline & NO & 447 & 441 & 461 & 506 & 1855 & & 297 & 272 & 309 \\
\hline & N/A & 0 & 3 & 0 & 2 & 5 & & 0 & 3 & 0 \\
\hline \multirow[t]{3}{*}{ Hypercholesterolemia } & YES & 467 & 472 & 466 & 432 & 1837 & \multirow[t]{3}{*}{0.3351} & 270 & 277 & 270 \\
\hline & NO & 464 & 461 & 465 & 491 & 1881 & & 302 & 294 & 300 \\
\hline & N/A & 9 & 6 & 7 & 12 & 34 & & 3 & 0 & 8 \\
\hline \multirow[t]{3}{*}{ Diabetes } & YES & 206 & 176 & 186 & 177 & 745 & \multirow[t]{3}{*}{0.3704} & 124 & 121 & 89 \\
\hline & NO & 702 & 723 & 717 & 708 & 2850 & & 429 & 431 & 463 \\
\hline & N/A & 32 & 40 & 35 & 50 & 157 & & 22 & 19 & 26 \\
\hline \multirow{3}{*}{$\begin{array}{l}\text { Difficulty walking or } \\
\text { climbing stairs }\end{array}$} & YES & 191 & 156 & 168 & 159 & 674 & \multirow[t]{3}{*}{0.1497} & 116 & 98 & 94 \\
\hline & NO & 748 & 783 & 770 & 775 & 3076 & & 459 & 473 & 484 \\
\hline & N/A & 1 & 0 & 0 & 1 & 2 & & 0 & 0 & 0 \\
\hline \multirow[t]{3}{*}{ Renal insufficiency } & YES & 54 & 40 & 41 & 42 & 177 & \multirow[t]{3}{*}{0.3868} & 28 & 16 & 21 \\
\hline & NO & 883 & 898 & 894 & 892 & 3567 & & 544 & 555 & 557 \\
\hline & N/A & 3 & 1 & 3 & 1 & 8 & & 3 & 0 & 0 \\
\hline \multirow[t]{3}{*}{ Asthma } & YES & 115 & 137 & 133 & 128 & 513 & \multirow[t]{3}{*}{0.4655} & 81 & 81 & 79 \\
\hline & NO & 825 & 801 & 804 & 807 & 3237 & & 494 & 490 & 499 \\
\hline & N/A & 0 & 1 & 1 & 0 & 2 & & 0 & 0 & 0 \\
\hline Arthritis & YES & 390 & 403 & 383 & 324 & 1500 & 0.0017 & 232 & 226 & 206 \\
\hline
\end{tabular}

Means with standard deviation (SD) were used for continuous characteristic variables and categorical variables were expressed as frequencies. Differences $\mathrm{i}$ used to analyze the differences of continuous variables between groups. In femur group: Q1:1.57-7.29 ug/L Q2:7.30-9.12 ug/L Q3:9.13-11.34 ug/L Q4:11.35body group: Q1:1.88-7.76 ug/L Q2:7.77-9.69 ug/L Q3:9.70-12.18 ug/L Q4:12.19-52.0 ug/L. 


\begin{tabular}{|c|c|c|c|c|c|c|c|c|c|c|}
\hline \multirow[t]{2}{*}{ Characteristic } & & \multicolumn{6}{|c|}{ Femur } & \multicolumn{3}{|c|}{ Spine } \\
\hline & & Q1 & Q2 & Q3 & Q4 & total & $P$ value & Q1 & Q2 & Q3 \\
\hline & NO & 547 & 535 & 553 & 606 & 2241 & & 343 & 345 & 371 \\
\hline & N/A & 3 & 1 & 2 & 5 & 11 & & 0 & 0 & 1 \\
\hline \multirow{3}{*}{$\begin{array}{l}\text { Congestive heart } \\
\text { failure }\end{array}$} & YES & 47 & 38 & 35 & 33 & 153 & \multirow[t]{3}{*}{0.3618} & 23 & 21 & 22 \\
\hline & NO & 884 & 899 & 901 & 900 & 3584 & & 546 & 552 & 556 \\
\hline & N/A & 9 & 2 & 2 & 2 & 15 & & 6 & 1 & 0 \\
\hline \multirow{3}{*}{$\begin{array}{l}\text { Coronary heart } \\
\text { disease }\end{array}$} & YES & 78 & 56 & 54 & 63 & 251 & \multirow[t]{3}{*}{0.111} & 44 & 25 & 22 \\
\hline & NO & 858 & 881 & 879 & 867 & 3485 & & 528 & 548 & 555 \\
\hline & N/A & 4 & 2 & 5 & 5 & 16 & & 3 & 1 & 1 \\
\hline \multirow[t]{3}{*}{ Angina pectoris } & YES & 38 & 42 & 31 & 31 & 142 & \multirow[t]{3}{*}{0.4572} & 22 & 22 & 15 \\
\hline & NO & 894 & 891 & 904 & 899 & 3588 & & 548 & 549 & 563 \\
\hline & N/A & 8 & 6 & 3 & 5 & 22 & & 5 & 3 & 0 \\
\hline \multirow[t]{3}{*}{ Storke } & YES & 73 & 48 & 46 & 41 & 208 & \multirow[t]{3}{*}{0.0064} & 46 & 28 & 25 \\
\hline & NO & 866 & 889 & 891 & 892 & 3538 & & 529 & 546 & 553 \\
\hline & N/A & 1 & 2 & 1 & 2 & 6 & & 0 & 0 & 0 \\
\hline \multirow[t]{3}{*}{ Thyroid problems } & YES & 115 & 140 & 146 & 161 & 562 & \multirow[t]{3}{*}{0.0240} & 70 & 81 & 86 \\
\hline & NO & 822 & 796 & 790 & 772 & 3180 & & 505 & 491 & 492 \\
\hline & N/A & 3 & 3 & 2 & 2 & 10 & & 0 & 2 & 0 \\
\hline \multirow[t]{3}{*}{ COPD } & YES & 62 & 65 & 62 & 49 & 238 & \multirow[t]{3}{*}{0.4417} & 35 & 26 & 39 \\
\hline & NO & 876 & 871 & 874 & 885 & 3506 & & 540 & 546 & 539 \\
\hline & N/A & 2 & 3 & 2 & 1 & 8 & & 0 & 2 & 0 \\
\hline \multirow[t]{3}{*}{ Smoking } & YES & 502 & 454 & 423 & 346 & 1725 & \multirow[t]{3}{*}{$<0.0001$} & 277 & 260 & 237 \\
\hline & NO & 438 & 485 & 515 & 589 & 2027 & & 298 & 314 & 341 \\
\hline & N/A & 0 & 0 & 0 & 0 & 0 & & 0 & 0 & 0 \\
\hline \multirow[t]{3}{*}{ Household smokers } & YES & 264 & 235 & 201 & 212 & 912 & \multirow[t]{3}{*}{0.0026} & 164 & 150 & 136 \\
\hline & NO & 643 & 672 & 709 & 702 & 2726 & & 395 & 404 & 427 \\
\hline & N/A & 33 & 32 & 28 & 21 & 114 & & 16 & 20 & 15 \\
\hline \multirow[t]{3}{*}{ Osteoporosis } & YES & 86 & 89 & 108 & 112 & 395 & \multirow[t]{3}{*}{0.1051} & 61 & 55 & 57 \\
\hline & NO & 852 & 845 & 824 & 819 & 3340 & & 513 & 516 & 515 \\
\hline & $\mathrm{N} / \mathrm{A}$ & 2 & 5 & 6 & 4 & 17 & & 1 & 3 & 6 \\
\hline
\end{tabular}

Means with standard deviation (SD) were used for continuous characteristic variables and categorical variables were expressed as frequencies. Differences $\mathrm{i}$ used to analyze the differences of continuous variables between groups. In femur group: Q1:1.57-7.29 ug/L Q2:7.30-9.12 ug/L Q3:9.13-11.34 ug/L Q4:11.35body group: Q1:1.88-7.76 ug/L Q2:7.77-9.69 ug/L Q3:9.70-12.18 ug/L Q4:12.19-52.0 ug/L.

\section{Result}

\section{Participant characteristics}

9732 subjects with available total body $(n=3682)$, femur $(n=3752)$, and spine $(n=2298)$ DEXA data were enrolled in this study. The average whole blood manganese level is $10.38 \pm 3.85 \mathrm{ug} / \mathrm{L}$ (range: 1.88 to $52.0 \mathrm{ug} / \mathrm{L}$ ) in total body DEXA data, $9.64 \pm 3.67 \mathrm{ug} / \mathrm{L}$ (range: 1.57 to $54.92 \mathrm{ug} / \mathrm{L}$ ) in femur DEXA data, $9.81 \pm 3.47 \mathrm{ug} / \mathrm{L}$ (range: 1.57 to $35.56 \mathrm{ug} / \mathrm{L}$ ) in spine DEXA data. Classified based on quartiles, the weighted characteristics of these participants are shown in Table 1 and Table S1. In the femur DEXA data group, the weighted mean age of the participants was $62.1 \pm 10.7$ years, $48.9 \%$ ( $n=1838$ ) were female, and there were significant differences in baseline characteristics among the groups in age, sex, race, body mass index, alcohol consumption, hypertension, osteoarthritis, stroke, thyroid problems, smoking, and family smoking (all $P<0.05$ ). In the spine DEXA data group, the weighted mean age of participants was $59.6 \pm 10.8$ years, $54.6 \%(n=1254)$ were female, and there were significant differences in baseline characteristics among the groups in age, sex, race, body mass index, alcohol consumption, hypertension, osteoarthritis, stroke, smoking status, diabetes, and family smoking (all P<0.05). In the total body DEXA data group, the weighted mean age of participants was $37.7 \pm 12.4$ years and $50.2 \%(n=1848)$ were female. There were significant differences in baseline characteristics among the groups in age, sex, race, body mass index, hypercholesterolemia, thyroid problems, smoking, and family smoking. 


\section{Relationship between blood manganese levels and bone mineral density}

Detailed results are represented in Table 2.1, 2.2, 2.3 and S2. Blood manganese levels are classified according to quartiles, the trends for blood manganese have statistical significance for left arm $\mathrm{BMD}(\mathrm{P}$ for trend < 0.01); left leg $\mathrm{BMD}(\mathrm{P}$ for trend $<0.01)$; right arm $\mathrm{BMD}(\mathrm{P}$ for trend $<0.01)$; right leg $\mathrm{BMD}(\mathrm{P}$ for trend < 0.01); left ribs $\mathrm{BMD}(\mathrm{P}$ for trend $<0.01)$; thoracic spine $\mathrm{BMD}(\mathrm{P}$ for trend $<0.05)$; lumber spine $\mathrm{BMD}(\mathrm{P}$ for trend $<0.01)$; pelvis $\mathrm{BMD}(\mathrm{P}$ for trend $<0.01)$; trunk bone $\mathrm{BMD}(\mathrm{P}$ for trend $<0.01)$; subtotal $\mathrm{BMD}(\mathrm{P}$ for trend $<0.01)$; total $\mathrm{BMD}(\mathrm{P}$ for trend $<0.01)$ in total body. 
Table 2.1

The relationship between blood manganese and BMD/BMC in femur

\begin{tabular}{|c|c|c|c|c|}
\hline & & Model 1 & Model 2 & Model 3 \\
\hline & & $\beta(95 \% \mathrm{Cl}) P$ value & $\beta(95 \% \mathrm{Cl}) P$ value & $\beta(95 \% \mathrm{Cl}) P$ value \\
\hline \multirow[t]{6}{*}{ Total femur BMD $(\mathrm{g} / \mathrm{cm} 2)$} & $\begin{array}{l}\text { Blood } \\
\text { manganese(ug/L) }\end{array}$ & $'-0.0615(-0.077,-0.046)<0.001$ & '-0.0174(-0.0298, -0.005)0.183 & $\begin{array}{l}-0.0224(-0.0361 \\
-0.0087) 0.122\end{array}$ \\
\hline & Q1:1.57-7.29 ug/L & Reference & Reference & Reference \\
\hline & Q2:7.30-9.12 ug/L & '-0.0151(-0.0589, 0.0287)0.743 & ‘0.0069(-0.0274, 0.0412)0.849 & '-0.005(-0.0421, 0.0321)0.898 \\
\hline & Q3:9.13-11.34 ug/L & $\begin{array}{l}-0.0514(-0.0952 \\
-0.0076) 0.265\end{array}$ & $\begin{array}{l}'-0.0031(-0.0375 \\
0.0313) 0.832\end{array}$ & $'-0.016(-0.0537,0.0217) 0.687$ \\
\hline & Q4:11.35-54.92 ug/L & $\begin{array}{l}'-0.155(-0.1988,-0.1112) \\
<0.001\end{array}$ & $\begin{array}{l}-0.0351(-0.0701 \\
-0.0001) 0.341\end{array}$ & '-0.0301(-0.069, 0.0088)0.463 \\
\hline & $P$ for trend & 0.004 & & \\
\hline \multirow[t]{6}{*}{ Total femur BMC(g) } & $\begin{array}{l}\text { Blood } \\
\text { manganese(ug/L) }\end{array}$ & $\begin{array}{l}'-0.162(-0.1773,-0.1467) \\
<0.001\end{array}$ & $\begin{array}{l}-0.0528(-0.063,-0.0426) \\
<0.001\end{array}$ & $\begin{array}{l}'-0.052(-0.0634,-0.0406) \\
<0.001\end{array}$ \\
\hline & Q1:1.57-7.29 ug/L & Reference & Reference & Reference \\
\hline & Q2:7.30-9.12 ug/L & $\begin{array}{l}'-0.1342(-0.1775 \\
-0.0909) 0.003\end{array}$ & $\begin{array}{l}-0.0435(-0.0717 \\
-0.0153) 0.143\end{array}$ & $\begin{array}{l}-0.0523(-0.0831 \\
-0.0215) 0.106\end{array}$ \\
\hline & Q3:9.13-11.34 ug/L & $\begin{array}{l}-0.2537(-0.297,-0.2104) \\
<0.001\end{array}$ & $\begin{array}{l}-0.0878(-0.1161, \\
-0.0595) 0.003\end{array}$ & $'-0.088(-0.1192,-0.0568) 0.007$ \\
\hline & Q4:11.35-54.92 ug/L & $\begin{array}{l}'-0.454(-0.4973,-0.4107) \\
<0.001\end{array}$ & $\begin{array}{l}-0.1467(-0.1755,-0.1179) \\
<0.001\end{array}$ & $\begin{array}{l}-0.134(-0.1663,-0.1017) \\
<0.001\end{array}$ \\
\hline & $P$ for trend & $<0.001$ & & \\
\hline \multirow[t]{6}{*}{$\begin{array}{l}\text { Femoral neck } \\
\mathrm{BMD}(\mathrm{g} / \mathrm{cm} 2)\end{array}$} & $\begin{array}{l}\text { Blood } \\
\text { manganese(ug/L) }\end{array}$ & $\begin{array}{l}-0.0526(-0.0681 \\
-0.0371) 0.001\end{array}$ & '-0.0361(-0.0495, -0.0227)0.01 & $\begin{array}{l}-0.0393(-0.0542, \\
-0.0244) 0.012\end{array}$ \\
\hline & Q1:1.57-7.29 ug/L & Reference & Reference & Reference \\
\hline & Q2:7.30-9.12 ug/L & '-0.0255(-0.0694, 0.0184)0.581 & $\begin{array}{l}-0.0223(-0.0595 \\
0.0149) 0.569\end{array}$ & $\begin{array}{l}-0.0451(-0.0853 \\
-0.0049) 0.286\end{array}$ \\
\hline & Q3:9.13-11.34 ug/L & $'-0.022(-0.0659,0.0219) 0.635$ & $\begin{array}{l}'-0.0118(-0.0491, \\
0.0255) 0.763\end{array}$ & $\begin{array}{l}-0.0167(-0.0575 \\
0.0241) 0.698\end{array}$ \\
\hline & Q4:11.35-54.92 ug/L & $\begin{array}{l}-0.1287(-0.1726, \\
-0.0848) 0.005\end{array}$ & $\begin{array}{l}-0.0847(-0.1226 \\
-0.0468) 0.034\end{array}$ & $\begin{array}{l}-0.0865(-0.1287 \\
-0.0443) 0.052\end{array}$ \\
\hline & $P$ for trend & 0.034 & & \\
\hline \multirow[t]{6}{*}{ Femoral neck BMC(g) } & $\begin{array}{l}\text { Blood } \\
\text { manganese(ug/L) }\end{array}$ & $\begin{array}{l}'-0.1241(-0.1395,-0.1087) \\
<0.001\end{array}$ & $\begin{array}{l}'-0.0544(-0.0665,-0.0423) \\
<0.001\end{array}$ & $'-0.0564(-0.07,-0.0428)<0.001$ \\
\hline & Q1:1.57-7.29 ug/L & Reference & Reference & Reference \\
\hline & Q2:7.30-9.12 ug/L & '-0.0794(-0.123, -0.0358)0.083 & $\begin{array}{l}'-0.0198(-0.0535, \\
0.0139) 0.577\end{array}$ & $\begin{array}{l}'-0.0324(-0.0691, \\
0.0043) 0.401\end{array}$ \\
\hline & Q3:9.13-11.34 ug/L & $\begin{array}{l}-0.1331(-0.1767 \\
-0.0895) 0.004\end{array}$ & '-0.0278(-0.0616, 0.006)0.434 & $'-0.0243(-0.0616,0.013) 0.535$ \\
\hline & Q4:11.35-54.92 ug/L & $\begin{array}{l}'-0.3397(-0.3833,-0.2961) \\
<0.001\end{array}$ & $\begin{array}{l}'-0.142(-0.1764,-0.1076) \\
<0.001\end{array}$ & $\begin{array}{l}'-0.1398(-0.1783,-0.1013) \\
<0.001\end{array}$ \\
\hline & $P$ for trend & $<0.001$ & & \\
\hline \multirow[t]{6}{*}{ Trochanter BMD(g/cm2) } & $\begin{array}{l}\text { Blood } \\
\text { manganese(ug/L) }\end{array}$ & $\begin{array}{l}-0.0443(-0.0598 \\
-0.0288) 0.007\end{array}$ & $\begin{array}{l}'-0.0056(-0.0187 \\
0.0075) 0.685\end{array}$ & $\begin{array}{l}-0.0126(-0.0271, \\
0.0019) 0.412\end{array}$ \\
\hline & Q1:1.57-7.29 ug/L & Reference & Reference & Reference \\
\hline & Q2:7.30-9.12 ug/L & '-0.0033(-0.0472, 0.0406)0.943 & ‘0.0143(-0.0219, 0.0505)0.707 & '0.0071(-0.0322, 0.0464)0.864 \\
\hline & Q3:9.13-11.34 ug/L & $'-0.0265(-0.0704,0.0174) 0.566$ & '0.0151(-0.0213, 0.0515)0.693 & ‘0.002(-0.0379, 0.0419)0.961 \\
\hline & Q4:11.35-54.92 ug/L & $\begin{array}{l}'-0.1045(-0.1484 \\
-0.0606) 0.024\end{array}$ & ‘0.0005(-0.0365, 0.0375)0.989 & '0.003(-0.0383, 0.0443)0.944 \\
\hline & $P$ for trend & 0.101 & & \\
\hline
\end{tabular}

Model 1: no covariates were adjusted. Model 2: age, sex, and race/ethnicity (Mexican American, other Hispanic, non-Hispanic White, non-Hispanic Black, other races) ,BMI were adjusted. Model 3: age, sex, race/ethnicity (Mexican American, other Hispanic, non-Hispanic White, non-Hispanic Black, other races), BMI, Drinking alcohol, Hypertension, Arthritis, Stroke, Thyroid problems, Smoking, Household smokers were adjusted. 


\begin{tabular}{|c|c|c|c|c|}
\hline & & Model 1 & Model 2 & Model 3 \\
\hline \multirow[t]{6}{*}{ Trochanter BMC(g) } & $\begin{array}{l}\text { Blood } \\
\text { manganese(ug/L) }\end{array}$ & $\begin{array}{l}-0.1481(-0.1634,-0.1328) \\
<0.001\end{array}$ & $\begin{array}{l}-0.0487(-0.0603,-0.0371) \\
<0.001\end{array}$ & $\begin{array}{l}-0.0528(-0.0659,-0.0397) \\
<0.001\end{array}$ \\
\hline & Q1:1.57-7.29 ug/L & Reference & Reference & Reference \\
\hline & Q2:7.30-9.12 ug/L & '-0.136(-0.1794, -0.0926) 0.003 & $'-0.0509(-0.083,-0.0188) 0.133$ & $\begin{array}{l}-0.0611(-0.0965 \\
-0.0257) 0.101\end{array}$ \\
\hline & Q3:9.13-11.34 ug/L & $\begin{array}{l}'-0.2302(-0.2736,-0.1868) \\
<0.001\end{array}$ & $\begin{array}{l}'-0.0756(-0.1078 \\
-0.0434) 0.026\end{array}$ & $\begin{array}{l}-0.0927(-0.1286 \\
-0.0568) 0.014\end{array}$ \\
\hline & Q4:11.35-54.92 ug/L & $\begin{array}{l}-0.4061(-0.4495,-0.3627) \\
<0.001\end{array}$ & $\begin{array}{l}'-0.1254(-0.1582,-0.0926) \\
<0.001\end{array}$ & '-0.1209(-0.158, -0.0838)0.002 \\
\hline & $P$ for trend & $<0.001$ & & \\
\hline \multirow[t]{6}{*}{$\begin{array}{l}\text { Intertrochanter } \\
\mathrm{BMD}(\mathrm{g} / \mathrm{cm} 2)\end{array}$} & $\begin{array}{l}\text { Blood } \\
\text { manganese(ug/L) }\end{array}$ & $-0.06(-0.0755,-0.0445)<0.001$ & $-0.0156(-0.0282,-0.03) 0.239$ & $-0.0217(-0.0357,-0.0077) 0.14$ \\
\hline & Q1:1.57-7.29 ug/L & Reference & Reference & Reference \\
\hline & Q2:7.30-9.12 ug/L & $-0.0138(-0.0576,0.03) 0.764$ & $0.0099(-0.0251,0.0449) 0.787$ & $-0.0023(-0.0404,0.0355) 0.953$ \\
\hline & Q3:9.13-11.34 ug/L & $-0.0528(-0.0966,-0.009) 0.253$ & $-0.003(-0.0381,0.0321) 0.936$ & $-0.0212(-0.0596,0.0172) 0.599$ \\
\hline & Q4:11.35-54.92 ug/L & $-0.154(-0.1978,-0.1102)<0.001$ & $-0.0331(-0.0688,0.0026) 0.379$ & $-0.0297(-0.0694,0.01) 0.477$ \\
\hline & $P$ for trend & 0.004 & & \\
\hline \multirow[t]{6}{*}{ Intertrochanter BMC(g) } & $\begin{array}{l}\text { Blood } \\
\text { manganese(ug/L) }\end{array}$ & $\begin{array}{l}-0.1614(-0.1767,-0.1461) \\
<0.001\end{array}$ & $\begin{array}{l}-0.0505(-0.0607,-0.0403) \\
<0.001\end{array}$ & $\begin{array}{l}-0.0476(-0.0591,-0.0361) \\
<0.001\end{array}$ \\
\hline & Q1:1.57-7.29 ug/L & Reference & Reference & Reference \\
\hline & Q2:7.30-9.12 ug/L & $-0.132(-0.1753,-0.0887) 0.004$ & $\begin{array}{l}-0.0411(-0.0694,-0.0128) \\
0.168\end{array}$ & $\begin{array}{l}-0.0483(-0.0793,-0.0173) \\
0.139\end{array}$ \\
\hline & Q3:9.13-11.34 ug/L & $\begin{array}{l}-0.2618(-0.3051,-0.2185) \\
<0.001\end{array}$ & $-0.0946(-0.123,-0.0662) 0.002$ & $-0.089(-0.1204,-0.0576) 0.007$ \\
\hline & Q4:11.35-54.92 ug/L & $\begin{array}{l}-0.4569(-0.5002,-0.4136) \\
<0.001\end{array}$ & $\begin{array}{l}-0.1454(-0.1743,-0.1165) \\
<0.001\end{array}$ & $\begin{array}{l}-0.1291(-0.1616,-0.0966) \\
<0.001\end{array}$ \\
\hline & $P$ for trend & $<0.001$ & & \\
\hline \multirow[t]{6}{*}{$\begin{array}{l}\text { Wards triangle } \\
\mathrm{BMD}(\mathrm{g} / \mathrm{cm} 2)\end{array}$} & $\begin{array}{l}\text { Blood } \\
\text { manganese(ug/L) }\end{array}$ & $0.0061(-0.0094,0.0216) 0.71$ & $-0.0191(-0.033,-0.0052) 0.193$ & $-0.0282(-0.0435,-0.0129) 0.08$ \\
\hline & Q1:1.57-7.29 ug/L & Reference & Reference & Reference \\
\hline & Q2:7.30-9.12 ug/L & $0.0337(-0.0102,0.0776) 0.466$ & $0.0068(-0.0319,0.0455) 0.868$ & $-0.0153(-0.0568,0.0262) 0.726$ \\
\hline & Q3:9.13-11.34 ug/L & $0.0954(0.0515,0.1393) 0.039$ & $0.0414(0.0026,0.0802) 0.311$ & $0.0211(-0.021,0.0632) 0.634$ \\
\hline & Q4:11.35-54.92 ug/L & $0.0461(0.0022,0.09) 0.319$ & $-0.0207(-0.0601,0.0187) 0.618$ & $-0.0265(-0.07,0.017) 0 . .564$ \\
\hline & $P$ for trend & 0.246 & & \\
\hline \multirow[t]{6}{*}{ Wards triangle $\mathrm{BMC}(\mathrm{g})$} & $\begin{array}{l}\text { Blood } \\
\text { manganese(ug/L) }\end{array}$ & $0.0119(-0.0036,0.0274) 0.465$ & $-0.0181(-0.0322,-0.004) 0.223$ & $\begin{array}{l}-0.0268(-0.0423,-0.0113) \\
0.102\end{array}$ \\
\hline & Q1:1.57-7.29 ug/L & Reference & Reference & Reference \\
\hline & Q2:7.30-9.12 ug/L & $0.0213(-0.0226,0.0652) 0.645$ & $\begin{array}{l}-0.0092(-0.0483,0.0299) \\
0.824\end{array}$ & $-0.0313(-0.0733,0.0107) 0.479$ \\
\hline & Q3:9.13-11.34 ug/L & $0.0897(0.0458,0.1336) 0.052$ & $0.0299(-0.0093,0.0691) 0.469$ & $0.0082(-0.0345,0.0509) 0.855$ \\
\hline & Q4:11.35-54.92 ug/L & $0.0589(0.015,0.1028) 0.202$ & $-0.0215(-0.0614,0.0184) 0.609$ & $-0.0231(-0.0672,0.021) 0.619$ \\
\hline & $P$ for trend & \multicolumn{3}{|l|}{0.237} \\
\hline $\begin{array}{l}\text { Model 1: no covariates } \\
\text { other races) ,BMI were a } \\
\text { BMI, Drinking alcohol, } \mathrm{H}\end{array}$ & $\begin{array}{l}\text { djusted. Model 2: age, } \\
\text { d. Model 3: age, sex, } \\
\text { nsion, Arthritis, Stroke }\end{array}$ & $\begin{array}{l}\text { and race/ethnicity (Mexican Ar } \\
\text { ethnicity (Mexican American, ot } \\
\text { roid problems, Smoking, House }\end{array}$ & $\begin{array}{l}\text { erican, other Hispanic, non-Hispar } \\
\text { er Hispanic, non-Hispanic White, } \\
\text { old smokers were adjusted. }\end{array}$ & $\begin{array}{l}\text { ic White, non-Hispanic Black, } \\
\text { on-Hispanic Black, other races), }\end{array}$ \\
\hline
\end{tabular}


Table 2.2

The relationship between blood manganese and BMD/BMC in spine

\begin{tabular}{|c|c|c|c|c|}
\hline & & Model 1 & Model 2 & Model 3 \\
\hline & & $\beta(95 \% \mathrm{Cl}) P$ value & $\beta(95 \% \mathrm{Cl}) P$ value & $\beta(95 \% \mathrm{Cl}) P$ value \\
\hline \multirow[t]{6}{*}{$\begin{array}{l}\text { Total spine } \\
\text { BMD }(\mathrm{g} / \mathrm{cm} 2)\end{array}$} & $\begin{array}{l}\text { Blood } \\
\text { manganese(ug/L) }\end{array}$ & $-0.0681(-0.0879,-0.0483) 0.001$ & $-0.0354(-0.0536,-0.0172) 0.007$ & $\begin{array}{l}-0.0251(-0.0458,-0.0044) \\
0.249\end{array}$ \\
\hline & Q1:1.57-7.27 ug/L & Reference & Reference & Reference \\
\hline & Q2:7.28-9.03 ug/L & $-0.0358(-0.0917,0.0201) 0.543$ & $-0.0316(-0.0822,0.019) 0.553$ & $\begin{array}{l}-0.0582(-0.1131,-0.0033) \\
0.314\end{array}$ \\
\hline & Q3:9.04-11.18 ug/L & $-0.0781(-0.1341,-0.0221) 0.185$ & $-0.0503(-0.1011,0.0005) 0.347$ & $-0.0157(-0.0715,0.0401) 0.789$ \\
\hline & Q4:11.19-35.56 ug/L & $-0.205(-0.2608,-0.1492)<0.001$ & $-0.1271(-0.1783,-0.0759) 0.018$ & $-0.0829(-0.14,-0.0258) 0.168$ \\
\hline & $P$ for trend & 0.003 & & \\
\hline \multirow[t]{6}{*}{ Total spine BMC(g) } & $\begin{array}{l}\text { Blood } \\
\text { manganese(ug/L) }\end{array}$ & $-0.1217(-0.1414,-0.102)<0.001$ & $-0.0541(-0.0712,-0.037) 0.003$ & $-0.0558(-0.0756,-0.036) 0.007$ \\
\hline & Q1:1.57-7.27 ug/L & Reference & Reference & Reference \\
\hline & Q2:7.28-9.03 ug/L & $-0.1622(-0.2178,-0.1066) 0.006$ & $-0.0867(-0.1342,-0.0392) 0.083$ & $-0.1071(-0.1596,-0.0546) 0.053$ \\
\hline & Q3:9.04-11.18 ug/L & $-0.2378(-0.2935,-0.1821)<0.001$ & $-0.1216(-0.1693,-0.0739) 0.015$ & $-0.0963(-0.1496,-0.043) 0.086$ \\
\hline & Q4:11.19-35.56 ug/L & $-0.3822(-0.4376,-0.3268)<0.001$ & $\begin{array}{l}-0.1946(-0.2427,-0.1465) \\
<0.001\end{array}$ & $\begin{array}{l}-0.1801(-0.2347,-0.1255) \\
0.002\end{array}$ \\
\hline & $P$ for trend & $<0.001$ & & \\
\hline \multirow[t]{6}{*}{ L1 BMD (g/cm2) } & $\begin{array}{l}\text { Blood } \\
\text { manganese(ug/L) }\end{array}$ & $-0.0534(-0.0732,-0.0336) 0.011$ & $-0.0208(-0.0388,-0.0028) 0.271$ & $-0.0068(-0.0271,0.0135) 0.751$ \\
\hline & Q1:1.57-7.27 ug/L & Reference & Reference & Reference \\
\hline & Q2:7.28-9.03 ug/L & $-0.0077(-0.0637,0.0483) 0.896$ & $-0.006(-0.0559,0.0439) 0.909$ & $-0.0384(-0.0924,0.0156) 0.499$ \\
\hline & Q3:9.04-11.18 ug/L & $-0.0412(-0.0973,0.0149) 0.485$ & $-0.016(-0.0661,0.0341) 0.762$ & $0.0174(-0.0376,0.0724) 0.764$ \\
\hline & Q4:11.19-35.56 ug/L & $-0.163(-0.2188,-0.1072) 0.006$ & $-0.0861(-0.1366,-0.0356) 0.106$ & $-0.0344(-0.0906,0.0218) 0.561$ \\
\hline & $P$ for trend & 0.0212 & & \\
\hline \multirow[t]{6}{*}{ L1 BMC(g) } & $\begin{array}{l}\text { Blood } \\
\text { manganese(ug/L) }\end{array}$ & $-0.1175(-0.1372,-0.0978)<0.001$ & $-0.0438(-0.0608,-0.0268) 0.014$ & $-0.0407(-0.0604,-0.021) 0.049$ \\
\hline & Q1:1.57-7.27 ug/L & Reference & Reference & Reference \\
\hline & Q2:7.28-9.03 ug/L & $-0.1371(-0.1927,-0.0815) 0.019$ & $-0.0603(-0.1074,-0.0132) 0.224$ & $-0.088(-0.1402,-0.0358) 0.109$ \\
\hline & Q3:9.04-11.18 ug/L & $-0.2168(-0.2725,-0.1611)<0.001$ & $-0.0953(-0.1426,-0.048) 0.055$ & $\begin{array}{l}-0.0677(-0.1208,-0.0146) \\
0.226\end{array}$ \\
\hline & Q4:11.19-35.56 ug/L & $-0.354(-0.4095,-0.2985)<0.001$ & $-0.1518(-0.1995,-0.1041) 0.003$ & $\begin{array}{l}-0.1279(-0.1822,-0.0736) \\
0.025\end{array}$ \\
\hline & $P$ for trend & $<0.001$ & & \\
\hline \multirow[t]{6}{*}{ L2 BMD(g/cm2) } & $\begin{array}{l}\text { Blood } \\
\text { manganese(ug/L) }\end{array}$ & $-0.0574(-0.0772,-0.0376) 0.006$ & $-0.0264(-0.0446,-0.0082) 0.168$ & $-0.0115(-0.0321,0.0091) 0.595$ \\
\hline & Q1:1.57-7.27 ug/L & Reference & Reference & Reference \\
\hline & Q2:7.28-9.03 ug/L & $-0.0346(-0.0905,0.0213) 0.558$ & $-0.0309(-0.0814,0.0196) 0.561$ & $-0.0541(-0.1088,0.0006) 0.347$ \\
\hline & Q3:9.04-11.18 ug/L & $-0.0614(-0.1175,-0.0054) 0.298$ & $-0.0364(-0.0871,0.0143) 0.495$ & $0.0017(-0.0539,0.0573) 0.976$ \\
\hline & Q4:11.19-35.56 ug/L & $-0.1862(-0.242,-0.1304) 0.002$ & $-0.118(-0.1629,-0.0607) 0.038$ & $-0.0543(-0.1112,0.0026) 0.365$ \\
\hline & $P$ for trend & 0.0094 & & \\
\hline
\end{tabular}

Model 1: no covariates were adjusted. Model 2: age, sex, and race/ethnicity (Mexican American, other Hispanic, non-Hispanic White, non-Hispanic Black, other races),BMI were adjusted. Model 3: age, sex, race/ethnicity (Mexican American, other Hispanic, non-Hispanic White, non-Hispanic Black, other races), BMI, Drinking alcohol, Hypertension, Arthritis, Stroke, Smoking status, Diabetes, Household smokers were adjusted. 


\begin{tabular}{|c|c|c|c|c|}
\hline & & Model 1 & Model 2 & Model 3 \\
\hline \multirow[t]{6}{*}{ L2 BMC(g) } & $\begin{array}{l}\text { Blood } \\
\text { manganese(ug/L) }\end{array}$ & $-0.1135(-0.1332,-0.0938)<0.001$ & $-0.0433(-0.0603,-0.0263) 0.016$ & $\begin{array}{l}-0.0435(-0.0632,-0.0238) \\
0.036\end{array}$ \\
\hline & Q1:1.57-7.27 ug/L & Reference & Reference & Reference \\
\hline & Q2:7.28-9.03 ug/L & $-0.1428(-0.1984,-0.0872) 0.015$ & $-0.0654(-0.1126,-0.0182) 0.188$ & $\begin{array}{l}-0.0881(-0.1403,-0.0359) \\
0.109\end{array}$ \\
\hline & Q3:9.04-11.18 ug/L & $-0.2051(-0.2608,-0.1494)<0.001$ & $-0.0875(-0.1349,-0.0401) 0.079$ & $\begin{array}{l}-0.0554(-0.1085,-0.0023) \\
0.321\end{array}$ \\
\hline & Q4:11.19-35.56 ug/L & $-0.3648(-0.4203,-0.3093)<0.001$ & $\begin{array}{l}-0.1705(-0.2183,-0.1227) \\
<0.001\end{array}$ & $\begin{array}{l}-0.1542(-0.2085,-0.0999) \\
0.007\end{array}$ \\
\hline & $P$ for trend & $<0.001$ & & \\
\hline \multirow[t]{6}{*}{ L3 BMD (g/cm2) } & $\begin{array}{l}\text { Blood } \\
\text { manganese(ug/L) }\end{array}$ & $-0.0688(-0.0886,-0.049)<0.001$ & $-0.0416(-0.0602,-0.023) 0.034$ & $\begin{array}{l}-0.0311(-0.0524,-0.0098) \\
0.165\end{array}$ \\
\hline & Q1:1.57-7.27 ug/L & Reference & Reference & Reference \\
\hline & Q2:7.28-9.03 ug/L & $-0.0215(-0.0774,0.0344) 0.715$ & $-0.0194(-0.0712,0.0324) 0.722$ & $-0.041(-0.0976,0.0156) 0.491$ \\
\hline & Q3:9.04-11.18 ug/L & $-0.0819(-0.1379,-0.0259) 0.165$ & $-0.0609(-0.1129,-0.0089) 0.266$ & $-0.0219(-0.0794,0.0356) 0.717$ \\
\hline & Q4:11.19-35.56 ug/L & $-0.1933(-0.2491,-0.1375) 0.001$ & $-0.1294(-0.1818,-0.077) 0.019$ & $\begin{array}{l}-0.0815(-0.1404,-0.0226) \\
0.188\end{array}$ \\
\hline & $P$ for trend & 0.0042 & & \\
\hline \multirow[t]{6}{*}{ L3 BMC(g) } & $\begin{array}{l}\text { Blood } \\
\text { manganese(ug/L) }\end{array}$ & $\begin{array}{l}-0.1226(-0.1423,-0.1029) \\
<0.001\end{array}$ & $-0.0591(-0.0766,-0.0416) 0.001$ & $-0.0602(-0.0804,-0.04) 0.005$ \\
\hline & Q1:1.57-7.27 ug/L & Reference & Reference & Reference \\
\hline & Q2:7.28-9.03 ug/L & $-0.1273(-0.1829,-0.0717) 0.029$ & $-0.0532(-0.1017,-0.0047) 0.297$ & $\begin{array}{l}-0.0715(-0.1252,-0.0178) \\
0.206\end{array}$ \\
\hline & Q3:9.04-11.18 ug/L & $-0.23(-0.2857,-0.1743)<0.001$ & $-0.1192(-0.1679,-0.0705) 0.02$ & $\begin{array}{l}-0.1008(-0.1554,-0.0462) \\
0.079\end{array}$ \\
\hline & Q4:11.19-35.56 ug/L & $-0.3729(-0.4283,-0.3175)<0.001$ & $-0.1959(-0.245,-0.1468)<0.001$ & $\begin{array}{l}-0.1782(-0.2341,-0.1223) \\
0.002\end{array}$ \\
\hline & $P$ for trend & $<0.001$ & & \\
\hline \multirow[t]{6}{*}{ L4 BMD (g/cm2) } & $\begin{array}{l}\text { Blood } \\
\text { manganese(ug/L) }\end{array}$ & $-0.0778(-0.0976,-0.058)<0.001$ & $-0.0391(-0.0575,-0.0207) 0.044$ & $\begin{array}{l}-0.0352(-0.0563,-0.0141) \\
0.114\end{array}$ \\
\hline & Q1:1.57-7.27 ug/L & Reference & Reference & Reference \\
\hline & Q2:7.28-9.03 ug/L & $-0.0608(-0.1167,-0.0049) 0.302$ & $-0.0513(-0.1024,-0.0002) 0.341$ & $\begin{array}{l}-0.0797(-0.1358,-0.0236) \\
0.177\end{array}$ \\
\hline & Q3:9.04-11.18 ug/L & $-0.0963(-0.1523,-0.0403) 0.102$ & $-0.0574(-0.1087,-0.0061) 0.288$ & $-0.0316(-0.0886,0.0254) 0.599$ \\
\hline & Q4:11.19-35.56 ug/L & $\begin{array}{l}-0.02186(-0.2744,-0.1628) \\
<0.001\end{array}$ & $-0.1251(-0.1769,-0.0733) 0.022$ & $-0.1003(-0.1586,-0.042) 0.102$ \\
\hline & $P$ for trend & 0.002 & & \\
\hline \multirow[t]{3}{*}{ L4 BMC(g) } & $\begin{array}{l}\text { Blood } \\
\text { manganese(ug/L) }\end{array}$ & $-0.1161(-0.1358,-0.0964)<0.001$ & $-0.0478(-0.0652,-0.0304) 0.009$ & $\begin{array}{l}-0.0528(-0.0731,-0.0325) \\
0.014\end{array}$ \\
\hline & Q1:1.57-7.27 ug/L & Reference & Reference & Reference \\
\hline & Q2:7.28-9.03 ug/L & $-0.1937(-0.2493,-0.1381)<0.001$ & $-0.1243(-0.1727,-0.0759) 0.015$ & $\begin{array}{l}-0.1472(-0.2012,-0.0932) \\
0.009\end{array}$ \\
\hline
\end{tabular}




\begin{tabular}{|c|c|c|c|}
\hline & Model 1 & Model 2 & Model 3 \\
\hline Q3:9.04-11.18 ug/L & $-0.23(-0.2857,-0.1743)<0.001$ & $-0.1155(-0.164,-0.067) 0.024$ & $\begin{array}{l}-0.0964(-0.1513,-0.0415) \\
0.096\end{array}$ \\
\hline Q4:11.19-35.56 ug/L & $-0.3677(-0.4232,-0.3122)<0.001$ & $\begin{array}{l}-0.1812(-0.2302,-0.1322) \\
<0.001\end{array}$ & $-0.179(-0.2352,-0.1228) 0.003$ \\
\hline$P$ for trend & $<0.001$ & & \\
\hline
\end{tabular}


Table 2.3

The relationship between blood manganese and BMD/BMC in total body

\begin{tabular}{|c|c|c|c|c|}
\hline & & Model 1 & Model 2 & Model 3 \\
\hline & & $\beta(95 \% \mathrm{Cl}) P$ value & $\beta(95 \% \mathrm{Cl}) P$ value & $\beta(95 \% \mathrm{Cl}) P$ value \\
\hline \multirow[t]{6}{*}{ Head BMD (g/cm2) } & $\begin{array}{l}\text { Blood } \\
\text { manganese(ug/L) }\end{array}$ & $0.0165(0.0008,0.0322) 0.318$ & $-0.053(-0.0684,-0.0376) 0.001$ & $-0.0593(-0.0775,-0.0411) 0.002$ \\
\hline & Q1:1.88-7.76 ug/L & Reference & Reference & Reference \\
\hline & Q2:7.77-9.69 ug/L & $-0.0824(-0.1266,-0.0382) 0.077$ & $-0.028(-0.0659,0.0099) 0.484$ & $-0.1261(-0.174,-0.0782) 0.012$ \\
\hline & Q3:9.70-12.18 ug/L & $-0.1396(-0.1838,-0.0954) 0.003$ & $0.0123(-0.0257,0.0503) 0.76$ & $\begin{array}{l}-0.2033(-0.2514,-0.1552) \\
<0.001\end{array}$ \\
\hline & Q4:12.19-52.0 ug/L & $-1808(-0.225,-0.1366)<0.001$ & $0.0641(0.0252,0.103) 0.118$ & $\begin{array}{l}-0.1984(-0.2484,-0.1484) \\
<0.001\end{array}$ \\
\hline & $P$ for trend & 0.0799 & & \\
\hline \multirow[t]{6}{*}{ Head BMC(g) } & $\begin{array}{l}\text { Blood } \\
\text { manganese(ug/L) }\end{array}$ & $\begin{array}{l}-0.01685(-0.0326, \\
-0.0012) 0.307\end{array}$ & $'-0.0348(-0.0658,-0.0187) 0.04$ & '-0.0468(-0.0658, -0.0278)0.019 \\
\hline & Q1:1.88-7.76 ug/L & Reference & Reference & Reference \\
\hline & Q2:7.77-9.69 ug/L & $\begin{array}{l}'-0.1346(-0.1789, \\
-0.0903) 0.004\end{array}$ & '-0.1471(-0.1911, -0.1031)0.002 & $'-0.1109(-0.1609,-0.0609) 0.035$ \\
\hline & Q3:9.70-12.18 ug/L & $\begin{array}{l}'-0.1119(-0.1561 \\
-0.0677) 0.016\end{array}$ & $'-0.1336(-0.1777,-0.0895) 0.004$ & $\begin{array}{l}'-0.1831(-0.2333,-0.1329) \\
<0.001\end{array}$ \\
\hline & Q4:12.19-52.0 ug/L & $\begin{array}{l}-0.0993(-0.1435 \\
-0.0551) 0.033\end{array}$ & '-0.1488(-0.1939, -0.1037)0.002 & $'-0.1628(-0.215,-0.1106) 0.003$ \\
\hline & $P$ for trend & 0.02 & & \\
\hline \multirow[t]{6}{*}{ Left Arm BMD(g/cm2) } & $\begin{array}{l}\text { Blood } \\
\text { manganese(ug/L) }\end{array}$ & $\begin{array}{l}-0.2316(-0.2468,-0.2164) \\
<0.001\end{array}$ & $\begin{array}{l}-0.0759(-0.0872,-0.0646) \\
<0.001\end{array}$ & $\begin{array}{l}-0.0665(-0.0799,-0.0531) \\
<0.001\end{array}$ \\
\hline & Q1:1.88-7.76 ug/L & Reference & Reference & Reference \\
\hline & Q2:7.77-9.69 ug/L & $-0.1406(-0.1838,-0.0974) 0.002$ & $-0.0737(-0.1046,-0.0428) 0.023$ & $-0.0162(-0.0514,0.019) 0.662$ \\
\hline & Q3:9.70-12.18 ug/L & $\begin{array}{l}-0.2937(-0.3368,-0.2506) \\
<0.001\end{array}$ & $\begin{array}{l}-0.1378(-0.1688,-0.1068) \\
<0.001\end{array}$ & $-0.121(-0.1563,-0.0859) 0.001$ \\
\hline & Q4:12.19-52.0 ug/L & $-0.604(-0.6472,-0.5608)<0.001$ & $\begin{array}{l}-0.2169(-0.2486,-0.1852) \\
<0.001\end{array}$ & $-0.1807(-0.2174,-0.144)<0.001$ \\
\hline & $P$ for trend & $\varangle 0.001^{\star \star \star}$ & & \\
\hline \multirow[t]{6}{*}{ Left Arm BMC(g) } & $\begin{array}{l}\text { Blood } \\
\text { manganese(ug/L) }\end{array}$ & $'-0.257(-0.2721,-0.2419)<0.001$ & $\begin{array}{l}'-0.1035(-0.1139,-0.0931) \\
<0.001\end{array}$ & $\begin{array}{l}'-0.0948(-0.1071,-0.0825) \\
<0.001\end{array}$ \\
\hline & Q1:1.88-7.76 ug/L & Reference & Reference & Reference \\
\hline & Q2:7.77-9.69 ug/L & $\begin{array}{l}'-0.1686(-0.2114,-0.1258) \\
<0.001\end{array}$ & $\begin{array}{l}'-0.1071(-0.1354,-0.0788) \\
<0.001\end{array}$ & $'-0.0755(-0.1079,-0.0431) 0.027$ \\
\hline & Q3:9.70-12.18 ug/L & $\begin{array}{l}'-0.3339(-0.3767,-0.2912) \\
<0.001\end{array}$ & $\begin{array}{l}'-0.1923(-0.2207,-0.1639) \\
<0.001\end{array}$ & $\begin{array}{l}'-0.1783(-0.2108,-0.1458) \\
<0.001\end{array}$ \\
\hline & Q4:12.19-52.0 ug/L & $\begin{array}{l}-0.7031(-0.7459,-0.6603) \\
<0.001\end{array}$ & $\begin{array}{l}'-0.3265(-0.3555,-0.2975) \\
<0.001\end{array}$ & $\begin{array}{l}'-0.2963(-0.3301,-0.2625) \\
<0.001\end{array}$ \\
\hline & $P$ for trend & $<0.001$ & & \\
\hline \multirow[t]{6}{*}{ Left Leg BMD(g/cm2) } & $\begin{array}{l}\text { Blood } \\
\text { manganese(ug/L) }\end{array}$ & $\begin{array}{l}-0.1881(-0.2035,-0.1727) \\
<0.001\end{array}$ & $\begin{array}{l}-0.0799(-0.0929,-0.0669) \\
<0.001\end{array}$ & $\begin{array}{l}-0.0698(-0.0852,-0.0544) \\
<0.001\end{array}$ \\
\hline & Q1:1.88-7.76 ug/L & Reference & Reference & Reference \\
\hline & Q2:7.77-9.69 ug/L & $-0.1032(-0.1468,-0.0596) 0.025$ & $-0.0608(-0.0964,-0.0252) 0.105$ & $-0.0088(-0.0492,0.0316) 0.837$ \\
\hline & Q3:9.70-12.18 ug/L & $\begin{array}{l}-0.2345(-0.2781,-0.1909) \\
<0.001\end{array}$ & $\begin{array}{l}-0.1316(-0.1673,-0.0959) \\
<0.001\end{array}$ & $-0.1304(-0.171,-0.0898) 0.002$ \\
\hline & Q4:12.19-52.0 ug/L & $\begin{array}{l}-0.4773(-0.5209,-0.4337) \\
<0.001\end{array}$ & $\begin{array}{l}-0.2073(-0.2439,-0.1707) \\
<0.001\end{array}$ & $-0.1803(-0.225,-0.1381)<0.001$ \\
\hline & $P$ for trend & 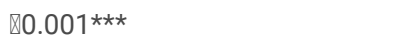 & & \\
\hline
\end{tabular}

Model 1: no covariates were adjusted. Model 2: age, sex, and race/ethnicity (Mexican American, other Hispanic, non-Hispanic White, non-Hispanic Black, other races), BMI were adjusted. Model 3: age, sex, race/ethnicity (Mexican American, other Hispanic, non-Hispanic White, non-Hispanic Black, other races), BMI, Hypercholesterolemia, Thyroid problems, Smoking, Household smokers were adjusted. 


\begin{tabular}{|c|c|c|c|c|}
\hline & & Model 1 & Model 2 & Model 3 \\
\hline \multirow[t]{6}{*}{ Left Leg BMC(g) } & $\begin{array}{l}\text { Blood } \\
\text { manganese(ug/L) }\end{array}$ & $\begin{array}{l}'-0.2421(-0.2573,-0.2269) \\
<0.001\end{array}$ & $\begin{array}{l}'-0.1068(-0.1183,-0.0953) \\
<0.001\end{array}$ & $\begin{array}{l}'-0.1048(-0.1184,-0.0912) \\
<0.001\end{array}$ \\
\hline & Q1:1.88-7.76 ug/L & Reference & Reference & Reference \\
\hline & Q2:7.77-9.69 ug/L & $\begin{array}{l}-0.1491(-0.1921 \\
-0.1061) 0.001\end{array}$ & $'-0.094(-0.1255,-0.0625) 0.005$ & 0.16 \\
\hline & Q3:9.70-12.18 ug/L & $\begin{array}{l}'-0.2977(-0.3407,-0.2547) \\
<0.001\end{array}$ & $\begin{array}{l}'-0.1661(-0.1977,-0.1345) \\
<0.001\end{array}$ & $<0.001$ \\
\hline & Q4:12.19-52.0 ug/L & $\begin{array}{l}'-0.6524(-0.6954,-0.6094) \\
<0.001\end{array}$ & $'-0.318(-0.3503,-0.2857)<0.001$ & $<0.001$ \\
\hline & $P$ for trend & $<0.001$ & & \\
\hline \multirow[t]{6}{*}{ Pelvis BMD(g/cm2) } & $\begin{array}{l}\text { Blood } \\
\text { manganese(ug/L) }\end{array}$ & $-0.0816(-0.066,-0.0972)<0.001$ & $-0.0515(-0.067,-0.036) 0.002$ & $-0.0439(-0.0623,-0.0255) 0.023$ \\
\hline & Q1:1.88-7.76 ug/L & Reference & Reference & Reference \\
\hline & Q2:7.77-9.69 ug/L & $-0.0608(-0.105,-0.0166) 0.191$ & $-0.0601(-0.1024,-0.0178) 0.178$ & $0.0046(-0.0438,0.053) 0.927$ \\
\hline & Q3:9.70-12.18 ug/L & $-0.0686(-0.1127,-0.0245) 0.139$ & $-0.0619(-0.1044,-0.0194) 0.166$ & $-0.0662(-0.1148,-0.0176) 0.196$ \\
\hline & Q4:12.19-52.0 ug/L & $\begin{array}{l}-0.2266(-0.2707,-0.1825) \\
<0.001\end{array}$ & $-0.1503(-0.1937,-0.1069) 0.001$ & $-0.1366(-0.1871,-0.0861) 0.01$ \\
\hline & $P$ for trend & $\nabla 0.001 \star \star \star$ & & \\
\hline \multirow[t]{6}{*}{ Pelvis BMC(g) } & $\begin{array}{l}\text { Blood } \\
\text { manganese(ug/L) }\end{array}$ & $\begin{array}{l}'-0.1768(-0.1922,-0.1614) \\
<0.001\end{array}$ & $\begin{array}{l}'-0.1042(-0.1183,-0.0901) \\
<0.001\end{array}$ & $\begin{array}{l}-0.1083(-0.1249,-0.0917) \\
<0.001\end{array}$ \\
\hline & Q1:1.88-7.76 ug/L & Reference & Reference & Reference \\
\hline & Q2:7.77-9.69 ug/L & $'-0.135(-0.1786,-0.0914) 0.003$ & $'-0.1133(-0.1519,-0.0747) 0.005$ & '-0.0639(-0.1075, -0.0203)0.164 \\
\hline & Q3:9.70-12.18 ug/L & $\begin{array}{l}-0.2222(-0.2658,-0.1786) \\
<0.001\end{array}$ & $\begin{array}{l}'-0.1462(-0.1849,-0.1075) \\
<0.001\end{array}$ & $'-0.136(-0.1798,-0.0922) 0.003$ \\
\hline & Q4:12.19-52.0 ug/L & $\begin{array}{l}'-0.5003(-0.5439,-0.4567) \\
<0.001\end{array}$ & $\begin{array}{l}'-0.3168(-0.3564,-0.2772) \\
<0.001\end{array}$ & $\begin{array}{l}-0.3146(-0.3601,-0.2691) \\
<0.001\end{array}$ \\
\hline & $P$ for trend & $<0.001$ & & \\
\hline \multirow[t]{6}{*}{$\begin{array}{l}\text { Trunk Bone } \\
\text { BMD }(\mathrm{g} / \mathrm{cm} 2)\end{array}$} & $\begin{array}{l}\text { Blood } \\
\text { manganese(ug/L) }\end{array}$ & $\begin{array}{l}-0.1324(-0.1479,-0.1169) \\
<0.001\end{array}$ & $-0.0872(-0.1024,-0.072)<0.001$ & $-0.0859(-0.104,-0.0679)<0.001$ \\
\hline & Q1:1.88-7.76 ug/L & Reference & Reference & Reference \\
\hline & Q2:7.77-9.69 ug/L & $-0.1022(-0.1462,-0.0582) 0.027$ & $-0.0919(-0.1336,-0.0502) 0.036$ & $-0.0383(-0.0859,0.0093) 0.44$ \\
\hline & Q3:9.70-12.18 ug/L & $\begin{array}{l}-0.1748(-0.2187,-0.1309) \\
<0.001\end{array}$ & $-0.1372(-0.179,-0.0954) 0.002$ & $-0.1458(-0.1936,-0.098) 0.004$ \\
\hline & Q4:12.19-52.0 ug/L & $\begin{array}{l}-0.3645(-0.4084,-0.3206) \\
<0.001\end{array}$ & $\begin{array}{l}-0.2504(-0.2931,-0.2077) \\
<0.001\end{array}$ & $\begin{array}{l}-0.2393(-0.2891,-0.1895) \\
<0.001\end{array}$ \\
\hline & $P$ for trend & $\varangle 0.001 * \star \star$ & & \\
\hline \multirow[t]{6}{*}{ Trunk Bone BMC(g) } & $\begin{array}{l}\text { Blood } \\
\text { manganese(ug/L) }\end{array}$ & $-0.1706(-0.186,-0.1552) \llbracket 0.001$ & $-0.0819(-0.0952,-0.0686) \otimes 0.001$ & $-0.0852(-0.1009,-0.0695) \llbracket 0.001$ \\
\hline & Q1:1.88-7.76 ug/L & Reference & Reference & Reference \\
\hline & Q2:7.77-9.69 ug/L & $-0.1012(-0.1448,-0.0576) 0.028$ & $-0.0777(-0.1139,-0.0415) 0.042$ & $-0.0329(-0.0743,0.0085) 0.449$ \\
\hline & Q3:9.70-12.18 ug/L & $-0.1884(-0.232,-0.1448)<0.001$ & $-0.1221(-0.1584,-0.0858) 0.001$ & $-0.1217(-0.1632,-0.0802) 0.005$ \\
\hline & Q4:12.19-52.0 ug/L & $\begin{array}{l}-0.4775(-0.5211,-0.4339) \\
<0.001\end{array}$ & $-0.2583(-0.2946,-0.222)<0.001$ & $\begin{array}{l}-0.2484(-0.2916,-0.2052) \\
<0.001\end{array}$ \\
\hline & $P$ for trend & $<0.001$ & & \\
\hline \multirow[t]{3}{*}{$\begin{array}{l}\text { Total body } \\
\text { BMD(g/cm2) }\end{array}$} & $\begin{array}{l}\text { Blood } \\
\text { manganese(ug/L) }\end{array}$ & $\begin{array}{l}-0.1409(-0.1564,-0.1254) \\
<0.001\end{array}$ & $\begin{array}{l}-0.0823(-0.0974,-0.0672) \\
<0.001\end{array}$ & $\begin{array}{l}-0.0789(-0.0968,-0.0610) \\
<0.001\end{array}$ \\
\hline & Q1:1.88-7.76 ug/L & Reference & Reference & Reference \\
\hline & Q2:7.77-9.69 ug/L & $-0.1365(-0.1804,-0.0926) 0.003$ & $-0.1168(-0.158,-0.0756) 0.007$ & $-0.0523(-0.0993,-0.0053) 0.29$ \\
\hline
\end{tabular}

Model 1: no covariates were adjusted. Model 2: age, sex, and race/ethnicity (Mexican American, other Hispanic, non-Hispanic White, non-Hispanic Black, other races), BMI were adjusted. Model 3: age, sex, race/ethnicity (Mexican American, other Hispanic, non-Hispanic White, non-Hispanic Black, other races), BMI, Hypercholesterolemia, Thyroid problems, Smoking, Household smokers were adjusted. 


\begin{tabular}{|c|c|c|c|c|}
\hline & & Model 1 & Model 2 & Model 3 \\
\hline & Q3:9.70-12.18 ug/L & $-0.2172(-0.261,-0.1734)<0.001$ & $\begin{array}{l}-0.1641(-0.2054,-0.1228) \\
<0.001\end{array}$ & $\begin{array}{l}-0.1826(-0.2298,-0.1354) \\
<0.001\end{array}$ \\
\hline & Q4:12.19-52.0 ug/L & $\begin{array}{l}-0.3847(-0.4286,-0.3408) \\
<0.001\end{array}$ & $-0.2389(-0.281,-0.1967)<0.001$ & $\begin{array}{l}-0.2188(-0.2679,-0.1697) \\
<0.001\end{array}$ \\
\hline & $P$ for trend & $\varangle 0.001^{\star \star \star}$ & & \\
\hline \multirow[t]{6}{*}{ Total body BMC(g) } & $\begin{array}{l}\text { Blood } \\
\text { manganese(ug/L) }\end{array}$ & $'-0.217(-0.2323,-0.2017)<0.001$ & $\begin{array}{l}'-0.1022(-0.1146,-0.0898) \\
<0.001\end{array}$ & $\begin{array}{l}-0.1025(-0.1172,-0.0878) \\
<0.001\end{array}$ \\
\hline & Q1:1.88-7.76 ug/L & Reference & Reference & Reference \\
\hline & Q2:7.77-9.69 ug/L & $\begin{array}{l}'-0.1606(-0.2038,-0.1174) \\
<0.001\end{array}$ & $'-0.1189(-0.1528,-0.085)<0.001$ & $'-0.0717(-0.1104,-0.033) 0.078$ \\
\hline & Q3:9.70-12.18 ug/L & $\begin{array}{l}'-0.2823(-0.3255,-0.2391) \\
<0.001\end{array}$ & $\begin{array}{l}'-0.1796(-0.2136,-0.1456) \\
<0.001\end{array}$ & $\begin{array}{l}'-0.1784(-0.2173,-0.1395) \\
<0.001\end{array}$ \\
\hline & Q4:12.19-52.0 ug/L & $\begin{array}{l}-0.6033(-0.6465,-0.5601) \\
<0.001\end{array}$ & $\begin{array}{l}'-0.3211(-0.3558,-0.2864) \\
<0.001\end{array}$ & $\begin{array}{l}'-0.3068(-0.3472,-0.2664) \\
<0.001\end{array}$ \\
\hline & $P$ for trend & $<0.001$ & & \\
\hline
\end{tabular}

In femur area the trends for blood manganese are statistically significant for total femur BMD ( $P$ for trend $<0.01)$; femoral neck $B M D(P$ for trend $<0.05)$; intertrochanter BMD ( $P$ for trend $<0.01)$ and in lumbar spine area, the trends for blood manganese have statistical significance for total spine $B M D(P$ for trend $<0.01$ ); L1 BMD (P for trend < 0.05); L2 BMD ( for trend < 0.01); L3 BMD ( for trend < 0.01); L4 BMD ( for trend < 0.01).

Weighted univariate and multiple linear regression models are constructed (Table 2.1, 2.2, 2.3). In the unadjusted model (Model 1 ), there is a significant negative correlation between increased blood manganese concentration and BMD of femur, spine and total body region $[\mathrm{P}<0.05$ except for wards triangle $\mathrm{BMD}$ ( $\beta$ coefficient $0.0061 ; 95 \% \mathrm{Cl}-0.0094,0.0216)$ and head BMD $(\beta$ coefficient $0.0165 ; 95 \% \mathrm{Cl} 0.0008,0.0322)$ ]. After adjusting for all the relevant covariables simultaneously(Model 3), femoral neck BMD ( $\beta$ coefficient $-0.0393 ; 95 \% \mathrm{Cl}-0.0542,-0.0244)$; head $\mathrm{BMD}(\beta$ coefficient $-0.0593 ; 95 \% \mathrm{Cl}-0.0775,-0.0411)$; left arm $\mathrm{BMD}(\beta$ coefficient $-0.0665 ; 95 \% \mathrm{Cl}-0.0799,-0.0531)$; left leg BMD ( $\beta$ coefficient $-0.0698 ; 95 \% \mathrm{Cl}-0.0852,-0.0544)$; pelvis BMD $(\beta$ coefficient $-0.0439 ; 95 \% \mathrm{Cl}$ $-0.0623,-0.0255)$; trunk bone $\mathrm{BMD}(\beta$ coefficient $-0.0859 ; 95 \% \mathrm{Cl}-0.104,-0.0679)$; Total BMD $(\beta$ coefficient $-0.0789 ; 95 \% \mathrm{Cl}-0.0968,-0.0610)$, decrease with increasing manganese levels, but not the spinal areas.

\section{Relationship between blood manganese levels and bone mineral content}

Detailed results are represented in Table 2.1, 2.2, 2.3 and S2. Blood manganese levels are classified according to quartiles, the trends for blood manganese have statistical significance for all the BMC of femur, spine and total body region (all P for trend $<0.05$ ). Weighted univariate and multiple linear regression models are constructed (Table 2.1,2.2, 2.3). In model 1, there was a significant negative correlation between increased blood manganese concentration and $\mathrm{BMC}$ of femur, spine and total body region $[\mathrm{P}<0.05$ except for wards triangle $\mathrm{BMC}(\beta$ coefficient $0.0119 ; 95 \% \mathrm{Cl}-0.0036,0.0274)$ and head $\mathrm{BMC}(\beta$ coefficient $-0.01685 ; 95 \% \mathrm{Cl}-0.0326,-0.0012)]$. In model $3, \mathrm{BMC}$ of femur, spine and total body, except wards triangle $\mathrm{BMC}(\beta$ coefficient $-0.0268 ; 95 \% \mathrm{Cl}-0.0423,-0.0113 ; \mathrm{P}$ 0.102), all decrease with increasing manganese levels.

\section{Subgroup analysis}

Subgroup analyses after adjusted for all relevant covariates are shown in Tables 3.1, 3.2, and 3.3. Multiple regression analyses in the femoral region have showed that, stratified by sex, femoral neck BMD decreases with increased blood manganese (Femoral neck BMD: $\beta$ coefficient $-0.0544 ; 95 \% \mathrm{Cl}-0.076,-0.0328$; $P$ 0.017), and femoral neck BMC decreases with increased blood manganese in all gender groups ( $\beta$ coefficient $<0, P<0.05$ ). Results stratified by race have showed that there are no significant correlation between femoral neck BMD/BMC and blood manganese in all races. The results of stratification by age have showed that the BMD of femoral neck decreased with the increase of blood manganese in $50-59$ and $60-69$ years [ $(\beta$-coefficient $-0.0581 ; 95 \% \mathrm{Cl}-0.0857$, $-0.0305 ; \mathrm{P} 0.046) ;(\beta$-coefficient -0.0561 . 95\% Cl -0.0825, -0.0297; P 0.043)], and there is a negative correlation between $\mathrm{BMC}$ and blood manganese. 
Table 3.1 Association of blood manganese with Femoral neck BMD/BMC, stratified by sex, race/ethnicity and age.

\begin{tabular}{|c|c|c|}
\hline & Femoral neck BMD(g/cm2) & Femoral neck BMC(g) \\
\hline & $\beta \otimes 95 \% \mathrm{CI} P \mathrm{P}$ value & $\beta \otimes 95 \% \mathrm{ClaP}$ value \\
\hline \multicolumn{3}{|l|}{ Subgroup analysis stratified by sex } \\
\hline Men & $-0.0351(-0.0563,-0.0139) 0.115$ & $-0.0655(-0.0032,-0.1278) 0.004$ \\
\hline Women & $-0.0544(-0.076,-0.0328) 0.017$ & $-0.073(-0.0959,-0.0501) 0.003$ \\
\hline \multicolumn{3}{|c|}{ Subgroup analysis stratified by race/ethnicity } \\
\hline Mexican American & $-0.0229(-0.0681,0.0223) 0.631$ & $-0.0417(-0.0837,0.0003) 0.347$ \\
\hline Other Hispanic & $-0.0446(-0.0889,-0.0003) 0.339$ & $-0.0397(-0.0782,-0.0012) 0.329$ \\
\hline Non-Hispanic White & $0.0114(-0.0113,0.0341) 0.634$ & $-0.0102(-0.0307,0.0103) 0.637$ \\
\hline Non-Hispanic Black & $0.0349(0.0029,0.0669) 0.301$ & $0.0321(0.003,0.0612) 0.296$ \\
\hline Other Race - Including Multi-Racial & $-0.0391(-0.1305,0.0523) 0.685$ & $-0.0394(-0.1258,0.047) 0.666$ \\
\hline \multicolumn{3}{|l|}{ Subgroup analysis stratified by age } \\
\hline $40-49$ & $0.0534 \rrbracket-0.0024,0.1092 \rrbracket 0.364$ & $0.0244(-0.0254,0.0742) 0.642$ \\
\hline $50-59$ & $-0.0581(-0.0857,-0.0305) 0.046$ & $-0.0643(-0.0891,-0.0395) 0.014$ \\
\hline $60-69$ & $-0.0561(-0.0825,-0.0297) 0.043$ & $-0.0674(-0.0906,-0.0442) 0.006$ \\
\hline $70-80$ & $-0.0094(-0.0391,0.0203) 0.764$ & $-0.0448(-0.071,-0.0186) 0.105$ \\
\hline
\end{tabular}

Age, sex, race/ethnicity (Mexican American, other Hispanic, non-Hispanic White, non-Hispanic Black, other races), BMI, Drinking alcohol, Hypertension, Arthritis, Stroke, Thyroid problems, Smoking, Household smokers were adjusted.

In the subgroup analysis stratified by sex or race/ethnicity, the model is not adjusted for the stratification variable itself.

Table 3.2 Association of blood manganese with Total spine BMD/BMC, stratified by sex, race/ethnicity and age.

\begin{tabular}{lll}
\hline & Total spine BMD $(\mathrm{g} / \mathrm{cm} 2)$ & Total spine BMC(g) \\
\hline Subgroup analysis stratified by sex & $\beta \nabla 95 \%$ ClaP value & $\beta \nabla 95 \%$ ClaP value \\
\hline Men & & \\
\hline Women & $-0.043(-0.0728,-0.0132) 0.171$ & $-0.075(-0.1062,-0.0438) 0.022$ \\
\hline Subgroup analysis stratified by race/ethnicity & $-0.0186(-0.0471,0.0099) 0.536$ & $-0.057(-0.0881,-0.0259) 0.082$ \\
\hline Mexican American & & \\
\hline Other Hispanic & $0.0591(0.0018,0.1164) 0.328$ & $0.0139(-0.0387,0.0665) 0.802$ \\
\hline Non-Hispanic White & $0.0141(-0.0429,0.0711) 0.814$ & $0.0446(-0.0093,0.0985) 0.433$ \\
\hline Non-Hispanic Black & $0.0005(-0.0329,0.0339) 0.988$ & $-0.0366(-0.068,-0.0052) 0.269$ \\
\hline Other Race - Including Multi-Racial & $0.0653(0.0196,0.111) 0.175$ & $0.0348(-0.0088,0.0784) 0.449$ \\
\hline Subgroup analysis stratified by age & $-0.0827(-0.2273,0.0619) 0.591$ & $-0.0239(-0.1709,0.1231) 0.878$ \\
\hline $40-49$ & & \\
\hline $50-59$ & $0.1017(0.0413,0.1621) 0.111$ & $0.0204(-0.0385,0.0793) 0.742$ \\
\hline $60-69$ & $-0.0978(-0.1326,-0.063) 0.008$ & $-0.1021(-0.1358,-0.0684) 0.004$ \\
\hline $70-80$ & $0.0095(-0.0255,0.0445) 0.796$ & $-0.0375(-0.0711,-0.0039) 0.289$ \\
\hline
\end{tabular}

Age, sex, race/ethnicity (Mexican American, other Hispanic, non-Hispanic White, non-Hispanic Black, other races), BMI, Drinking alcohol, Hypertension, Arthritis, Stroke[MOU1], Smoking status, Diabetes, Household smokers were adjusted.

In the subgroup analysis stratified by sex or race/ethnicity, the model is not adjusted for the stratification variable itself. 
Table 3.3 Association of blood manganese with Total body BMD/BMC, stratified by sex, race/ethnicity and age.

\begin{tabular}{lll} 
& Total body BMD $(\mathrm{g} / \mathrm{cm} 2)$ & Total body BMC(g) \\
\hline Subgroup analysis stratified by sex & $\beta \nabla 95 \%$ CI P value & $\beta \otimes 95 \%$ ClaP value \\
\hline Men & & \\
\hline Women & $-0.1455(-0.1708,-0.1202)<0.001$ & $-0.1861(-0.2101,-0.1621)<0.001$ \\
\hline Subgroup analysis stratified by race/ethnicity & $-0.0324(-0.0584,-0.0064) 0.237$ & $-0.0674(-0.0919,-0.0429) 0.009$ \\
\hline Mexican American & $-0.0455(-0.0887,-0.0023) 0.319$ & $-0.1157(-0.1495,-0.0819) 0.001$ \\
\hline Other Hispanic & $0.0139(-0.0407,0.0685) 0.808$ & $0.0054(-0.0383,0.0491) 0.906$ \\
\hline Non-Hispanic White & $-0.0106(-0.0409,-0.0197) 0.74$ & $-0.0206(-0.0451,0.0039) 0.426$ \\
\hline Non-Hispanic Black & $0.0033(-0.0401,0.0467) 0.943$ & $0.0366(0.0001,0.0732) 0.343$ \\
\hline Other Race - Including Multi-Racial & $-0.1046(-0.1797,-0.0295) 0.188$ & $-0.073(-0.134,-0.012) 0.258$ \\
\hline Subgroup analysis stratified by age & & \\
\hline 18-29 & $-0.1255(-0.1604,-0.0906)<0.001$ & $-0.1402(-0.1687,-0.1117)<0.001$ \\
\hline $30-39$ & $-0.0883(-0.1238,-0.0528) 0.018$ & $-0.0769(-0.1059,-0.0479) 0.012$ \\
\hline $40-49$ & $-0.0361(-0.0761,0.0039) 0.391$ & $-0.1082(-0.1415,-0.0749) 0.002$ \\
\hline $50-59$ & $-0.0688(-0.1014,-0.0362) 0.046$ & $-0.0934(-0.1205,-0.0663) 0.001$ \\
\hline
\end{tabular}

Age, sex, race/ethnicity (Mexican American, other Hispanic, non-Hispanic White, non-Hispanic Black, other races), BMI, Hypercholesterolemia, Thyroid problems, Smoking, Household smokers were adjusted.

In the subgroup analysis stratified by sex or race/ethnicity, the model is not adjusted for the stratification variable itself.

\section{[MOU1]stroke}

In the spinal region, gender stratified results have showed that only the total spinal BMC is significantly correlated with the blood manganese, and no significant correlations are found between the total spinal BMD and the blood manganese in all gender groups. Results stratified by race have showed that there is no significant correlation between $\mathrm{BMD} / \mathrm{BMC}$ and blood manganese in all races. After stratified by age, BMD/BMC is negatively correlated with blood manganese in the total spine at $50-59$ years of age $[(\beta-$ coefficient $-0.0978 ; 95 \% \mathrm{Cl}-0.1326,-0.063 ; \mathrm{P} 0.008) ;(\beta-\operatorname{coefficient}-0.1021 ; 95 \% \mathrm{Cl}-0.1358,-0.0684 ; \mathrm{P}$ 0.004)].

In the total body region, after stratified by sex, BMD decreased significantly with blood manganese increasing in males ( $\beta$-coefficient -0.1455 ; $95 \% \mathrm{Cl}-0.1708$, -0.1202; $P<0.001$ ), while total body BMC decreased with blood manganese increasing in all gender groups $(\beta$ coefficient $<0, P<0.05)$. Results stratified by race have showed that there is no significant correlation between BMD/BMC of the total body and blood manganese in all races, except BMC in Mexican American ( $\beta$-coefficient $-0.1157 ; 95 \% \mathrm{Cl}-0.1495,-0.0819 ; \mathrm{P} 0.001$ ). Results stratified by age have showed a significant negative correlation in most age groups between 18 to 59 ( $\beta$ coefficient $<0, P<0.05)$, except $40-49$.

\section{Discussion}

The incidence of osteoporosis is rising, with the increasing longevity of global population. Osteoporotic fractures, also known as fragility fractures, are a major complication of osteoporosis, often occurring in the spine, hip, distal forearm, and proximal humerus. These fractures often lead to chronic pain, depression, disability, reduced life quality and increased mortality. The annual number of fragility fractures in the EU is projected to increase from 3.5 million in 2010 to 4.5 million in 2025, with a significant loss of quality-adjusted life years (QALYs) and a significant increase in economic burden ${ }^{1,12}$. The optimal use of fracture prevention interventions is therefore an important priority in health-care delivery. According to International Commission on Radiation Protection (ICRP), about $40 \%$ of body manganese accumulates in bones ${ }^{13}$. Thus we hypothesized that blood manganese might somehow be associated with BMD/BMC. The aim of this study is to assess the relationship between blood manganese and BMD/BMC using a representative sample from the National Health and Nutrition Examination Survey (NHANES). The study population is a large, nationally representative sample of Americans over the age of 18 . In contrast to previous studies, after adjusting for confounders, we found that BMD in the femoral neck and total body is negatively associated with blood manganese, while BMD in the spine is not significantly negatively associated with blood manganese. The BMC of femur, spine and total body has showed significant negative correlation with blood manganese. Subgroup analysis have showed that for BMD, blood manganese is negatively correlated with femur neck BMD in females aged between 50-70 years, spinal BMD in 50-60 years old, and total body BMD in males and most ages of 18-60 years old. For BMC, blood manganese is inversely associated with femur neck BMC in males or females, 50-70 years of age, spinal BMC in males, 50-60 years of age, and total body BMC in males or females, Mexican Americans, and 18-60 years of age.

Multiple linear regression analysis and subgroup analysis have showed that the relationship between blood manganese and BMD has mainly been found on the femoral neck and total body, but not the spine. This may be attributed to the different structure of the femur and spine, as previous studies shown, the trabecular bone of the lumbar spine is dominant compared to the femoral neck, which contains a higher proportion of cortical bone. Although trabecular bone 
makes up only $20 \%$ of bone mass in healthy adult bones, it has a greater surface area and remodeling rate than cortical bone ${ }^{14,15}$. However, due to the lack of research on the specific mechanism of blood manganese in bone metabolism, further basic research is necessary. Interestingly, it is noteworthy that no matter in femoral neck, spine or total body, blood manganese concentration is significantly negatively correlated with BMD/BMC in people between 50-60 years old, indicating that blood manganese concentration has certain predictive value for bone mineral density as well as bone mass in those between $50-60$ years old. The explanation for this phenomenon is unclear, however, owing to changes in physical function, age may be a common factor influencing an individual's susceptibility to manganese toxicity. In addition, our study has found that in the femoral neck, blood manganese is significantly negatively correlated with BMD in female instead of male, while in the total body, blood manganese is significantly negatively correlated with BMD in male rather than female. On the one way, it may account for this difference that the femur DEXA data focused on people over 40 years of age while the DEXA data on total body are concentrated in people aged 8-59, and the data under 18 years old are excluded in the study. On the other way, we speculate that different sex hormones levels may have different function on the BMD in femoral neck or total body.

Previous studies have shown that most changes in BMD, especially in the hip, can be attributed to BMC variation, and both are considered to be important determinants of osteoporotic fractures ${ }^{16}$. In our study, blood manganese is significantly negatively correlated with BMC in most regions of femur, spine and total body. It is consistent with previous finding that bone weight reduction was observed in rats with long-term oral manganese intake ${ }^{17}$. However, the association between blood manganese level and bone loss remained debatable, and some previous epidemiological studies have shown mixed findings. A study determining the effect of manganese $(\mathrm{Mn})$ supplementation on BMD and bone metabolic parameters in ovariectomized Sprague-Dawley rats found that manganese supplementation improved mineral density and serum osteocalcin in the spine and femur of rats ${ }^{18}$. Another prospective cross-sectional study of 41 untreated postmenopausal women showed a significant positive association between serum manganese levels and lumbar or femoral BMD ${ }^{19}$. Odabasi et al., in a case-control study of 138 postmenopausal women, reported that there was no significant differences in blood manganese levels between postmenopausal women with osteoporosis and postmenopausal women without osteoporosis. The median blood manganese concentration was 14.76 $\mathrm{ng} / \mathrm{mL}$ in the osteoporosis group and $15.54 \mathrm{ng} / \mathrm{mL}$ in the control group ${ }^{20}$. Wang et al found no correlation between plasma manganese and bone mineral density in an epidemiological study of 91 elderly men over 50 years old in Beijing ${ }^{21}$. However, in a cross-sectional study of 304 retired workers with occupational manganese exposure, aiming to investigate the relationship between long-term occupational manganese exposure and bone quality, female participants in the highest manganese exposure group were significantly lower than the female control group in terms of stiffness index (SI) and T-score levels. This suggests that women with occupational manganese exposure may have a higher risk of osteoporosis ${ }^{9}$. Different sample sizes, inclusion and exclusion criteria, duration of follow-up, and different levels of manganese exposure may lead to different results between studies. These results indicate that the relationship between blood manganese and bone mineral density is still uncertain, and further basic studies are needed to explore the relationship between manganese and bone metabolism.

Our study shows that blood manganese levels are associated with bone mineral density of femur and total body, and high blood manganese levels may affect bone metabolism. Bone is one of the body's main organs for long-term storage of manganese, and according to the International Commission on Radiation Protection (ICRP), about $40 \%$ of body manganese accumulates in bones ${ }^{13}$. Evidence from animal study suggested that Mn accumulated in rat bones with an average elimination half-life of 143 days, equivalent to approximately 8.5 years in human bones ${ }^{17}$. Thus bone may also be an ideal organ exploring toxicity which caused by long-term manganese exposure ${ }^{9}$. It has been previously reported that excessive manganese may be neurotoxic to humans, affecting specific areas of the central nervous system and producing irreversible neurological symptoms ${ }^{22}$. Occupational exposure concentrations of airborne manganese in the range of $0.1-1.27 \mathrm{mg} / \mathrm{m} 3$ indicate blood levels of $10.3-12.5 \mathrm{ug} / \mathrm{L}^{23}$. In patients with clinical signs and symptoms of manganese poisoning, blood manganese concentration varies between 4 and $40 \mathrm{ug} / \mathrm{L}^{17,24-26}$. Studies have shown that dietary manganese deficiency and excessive manganese exposure can increase the production of reactive oxygen species (ROS), and abnormal accumulation of excessive ROS will exacerbate oxidative stress and inflammation, which is crucial in the pathogenesis of metabolic diseases ${ }^{27}$. Recent studies ${ }^{28,29}$ have found that during osteoblast differentiation, mitochondrial biogenesis, mitochondrial function (especially the activity of complex I in mitochondrial ECT) and ATP content are significantly increased, while excessive manganese accumulation in mitochondria inhibits mitochondrial complex I and II respiration and induces permeability transition ${ }^{27,30-32}$. It may disrupt mitochondrial homeostasis and lead to mitochondrial dysfunction, ultimately adversely affecting BMD. In addition, proposed in recent years, mitophagy has been reported that it improves tissue homeostasis via recovering energy by limiting the energy demands of ineffective organelles, reducing intracellular ROS produced by impaired mitochondria, and generating ATP during degradation under physiological or pathological conditions, while the disorder of mitophagy damages cells energy metabolism and physiological function, and breaks mitochondrial homeostasis ${ }^{28,33}$. Thus we speculate that excess manganese may also adversely affect bone metabolism through the mitophagy pathway, and the mechanism of this still needs further studied.

Significantly higher blood Mn levels have been revealed in women of US residents, Canadians, Koreans, Chinese general population, and Italians ${ }^{9,34-38 . ~}$ Mining and iron as well as steel production are considered as occupational sources of manganese exposure. Humans are more likely to be exposed to manganese through diet. Nuts, grain products, chocolate, crustaceans, legumes, mollusks, fresh fruits and tea are considered as rich sources of manganese ${ }^{39}$. In the United States, the recommended daily intake is $1.8 \mathrm{mg} /$ day for women and $2.3 \mathrm{mg} /$ day for men ${ }^{40}$. Excessive intake of manganese has been reported to be associated with cognitive impairment in human 41,42 . According to the National Institutes of Health, consuming more than 11 milligrams a day may have bad effects and the typical non-vegetarian Western diets provide 3-7 mg of manganese per day ${ }^{43}$. Previous studies have suggested that routine supplementation of manganese and other metals is generally unnecessary and such excessive supplementation may be harmful ${ }^{40}$. In the current study, we reported a negative association between blood manganese in adults selected from NHANES in 2013-2014 and 2017-2018 and femur and total body $\mathrm{BMD} / \mathrm{BMC}$. As the results showed, manganese intake may remain as an important public health issue. Some multivitamins typically contain 2-4 mg of additional manganese, and those with long-term intake, especially older adults, need to pay extra attention to their long-term risks and benefits. While in Europe, the European Food Safety Agency (EFSA), considering lacking of the sufficient and definitive data concerning humans, has neither established any tolerable upper intake Level (UL), nor an average requirement (Average Requirement, AR) and a reference intake for the population (population reference intake,

Page $17 / 21$ 
PRI) for $\mathrm{Mn}^{44,45}$. The results of our study may provide some references for the establishment of AR, PRI and UL of Mn. In addition, regular dual-energy radiographs of the femur or total body are recommended for people exposed to manganese, including those living near factories where manganese is utilized in production, especially women, to be aware of the risk of osteoporosis.

The present study was the first to investigate the relationship between manganese status and BMD/BMC in a large nationally representative sample, adequately controlling for confounding factors, and stratified analysis by age, sex, and race to investigate the relationship between blood manganese status and BMD/BMC in different populations. This study still has some limitations. (i) As a cross-sectional study, the causal relationship between Mn and $\mathrm{BMD} / \mathrm{BMC}$ cannot be determined. On the one hand, if high manganese leads to the decrease of BMD / BMC, great attention should be paid to the influence of blood manganese level in daily diet and life. On the other hand, about $40 \%$ of manganese is accumulated in bone ${ }^{13}$. If the increase of blood manganese level is caused by the release of manganese from bone tissue after bone loss, the increase of blood manganese level may have potential predictive value for osteoporosis. (ii) The total body BMD and BMC in the NHANES database only included data from samples aged 8-59 years (we excluded data under 18 years old), while the BMD and BMC of femur and spine only included data over 40 years old, which could not properly represent the American population over 18 years old. (iii) The study only looked at people in the United States, and the results may not accurately apply to other countries or regions. (iv) Residual or unmeasured confounders are possible despite adjustment for potential known confounders.

\title{
Conclusion
}

This nationwide cross-sectional study has showed a negative association between blood manganese levels and femur as well as total body BMD/BMC, especially femoral neck BMD in women aged 50-70 years. People exposed to manganese should be aware of the increased risk of osteopenia or osteoporosis. Besides, due to the lack of available data, there are no definite values for the tolerable upper intake level (UL), average requirement (AR) and population reference intake (PRI) of manganese. The results of our study may provide some references for the establishment of AR, PRI and UL of Mn.

\section{Abbreviations}

\author{
NHANES \\ National Health and Nutrition Examination Survey \\ BMD \\ bone mineral density \\ $\mathrm{BMC}$ \\ bone mineral content \\ EFSA \\ European Food Safety Agency \\ UL \\ upper intake level \\ AR \\ average requirement \\ PRI \\ population reference intake \\ DEXA \\ Dual Energy X-ray Absorptiometry \\ QALYS \\ quality-adjusted life years \\ ICRP \\ International Commission on Radiation Protection \\ SI \\ stiffness index \\ ROS \\ reactive oxygen species
}

\section{Declarations}

\section{Ethics approval and consent to participate}

The studies involving human participants were reviewed and approved by National Center for Health Ethics Review Board. The patients/participants provided their written informed consent to participate in this study.

\section{Availability of data and materials}

Datasets of this study are all available at https://wwwn.cdc.gov/nchs/nhanes/. All the data are available if qualified authors apply for them.

\section{Competing interests}

The authors declare no competing interests. 


\section{Authors' Contributions}

WC and ZY contributed equally to this work. WC, ZY, and ZSS designed the study. ZSS performed the analyses. WC, ZY, ,OMN and ZSS analyzed data. WC, ZY and ZSS interpreted the data, wrote the manuscript and revised the manuscript. All authors approve of the final version of the manuscript.

\section{Funding}

This work was financially supported by the National Natural Science Foundation of China (Nos. 81902222) and the Natural Science Foundation of Hunan Province (2020JJ4928)

\section{Consent for publication}

Not applicable.

\section{References}

1. Johnston, C. B.; Dagar, M., Osteoporosis in Older Adults. The Medical clinics of North America 2020, 104 (5), 873-884.

2. Farrah, Z.; Jawad, A. S., Optimising the management of osteoporosis. Clinical medicine (London, England) 2020, 20 (5), e196-e201.

3. Looker, A.; Frenk, S. J. D. o. H.; Nutrition Examination Surveys, C., Percentage of adults aged 65 and over with osteoporosis or low bone mass at the femur neck or lumbar spine: United States, 2005-2010. 2015.

4. Chen, P.; Bornhorst, J.; Aschner, M., Manganese metabolism in humans. Frontiers in bioscience (Landmark edition) 2018, 23, 1655-1679.

5. Yoon, M.; Schroeter, J. D.; Nong, A.; Taylor, M. D.; Dorman, D. C.; Andersen, M. E.; Clewell, H. J., 3rd, Physiologically based pharmacokinetic modeling of fetal and neonatal manganese exposure in humans: describing manganese homeostasis during development. Toxicological sciences: an official journal of the Society of Toxicology 2011, 122 (2), 297-316.

6. Deng, Q.; Liu, J.; Li, Q.; Chen, K.; Liu, Z.; Shen, Y.; Niu, P.; Yang, Y.; Zou, Y.; Yang, X., Interaction of occupational manganese exposure and alcohol drinking aggravates the increase of liver enzyme concentrations from a cross-sectional study in China. Environmental health: a global access science source 2013, 12,30 .

7. Pejović-Milić, A.; Chettle, D. R.; Oudyk, J.; Pysklywec, M. W.; Haines, T., Bone manganese as a biomarker of manganese exposure: a feasibility study. American journal of industrial medicine 2009, 52 (10), 742-50.

8. Saltman, P. D.; Strause, L. G., The role of trace minerals in osteoporosis. Journal of the American College of Nutrition 1993, 12 (4), $384-9$.

9. Li, D.; Ge, X.; Liu, Z.; Huang, L.; Zhou, Y.; Liu, P.; Qin, L.; Lin, S.; Liu, C.; Hou, Q.; Li, L.; Cheng, H.; Ou, S.; Wei, F.; Shen, Y.; Zou, Y.; Yang, X., Association between long-term occupational manganese exposure and bone quality among retired workers. Environmental science and pollution research internationa/ 2020 , $27(1), 482-489$.

10. Zhou, J. J. J. o. H. R., Tolerable upper intake level on vitamins and minerals. 2004, 33 (6), 1467.

11. Zipf, G.; Chiappa, M.; Porter, K. S.; Ostchega, Y.; Lewis, B. G.; Dostal, J., National health and nutrition examination survey: plan and operations, $1999-2010$. Vital and health statistics. Ser. 1, Programs and collection procedures 2013, (56), 1-37.

12. Kanis, J. A.; Cooper, C.; Rizzoli, R.; Reginster, J. Y., European guidance for the diagnosis and management of osteoporosis in postmenopausal women. Osteoporosis international: a journal established as result of cooperation between the European Foundation for Osteoporosis and the National Osteoporosis Foundation of the USA 2019, 30 (1), 3-44.

13. Report of the task group on reference man. Annals of the ICRP1979, 3 (1-4), iii.

14. Karamati, M.; Yousefian-Sanni, M.; Shariati-Bafghi, S. E.; Rashidkhani, B. J. C. T. I., Major Nutrient Patterns and Bone Mineral Density among Postmenopausal Iranian Women. 2014, 94 (6), 648.

15. Compston, J. E., Osteoporosis. Clinical endocrinology 1990, 33 (5), 653-82.

16. Deng, H. W.; Xu, F. H.; Davies, K. M.; Heaney, R.; Recker, R. R., Differences in bone mineral density, bone mineral content, and bone areal size in fracturing and non-fracturing women, and their interrelationships at the spine and hip. Journal of bone and mineral metabolism 2002, 20 (6), 358-66.

17. O'Neal, S. L.; Hong, L.; Fu, S.; Jiang, W.; Jones, A.; Nie, L. H.; Zheng, W., Manganese accumulation in bone following chronic exposure in rats: steady-state concentration and half-life in bone. Toxicology letters 2014, 229 (1), 93-100.

18. Bae, Y. J.; Kim, M. H., Manganese supplementation improves mineral density of the spine and femur and serum osteocalcin in rats. Biological trace element research 2008, 124 (1), 28-34.

19. Němčíková, P.; Spěváčková, V.; Čejchanová, M.; Hill, M.; Žofková, l., Relationship of serum manganese and copper levels to bone density and quality in postmenopausal women. A pilot study. 2009, 14 (3), 97-100.

20. Odabasi, E.; Turan, M.; Aydin, A.; Akay, C.; Kutlu, M., Magnesium, zinc, copper, manganese, and selenium levels in postmenopausal women with osteoporosis. Can magnesium play a key role in osteoporosis? Annals of the Academy of Medicine, Singapore 2008, 37 (7), $564-7$.

21. Wang, L.; Yu, H.; Yang, G.; Zhang, Y.; Wang, W.; Su, T.; Ma, W.; Yang, F.; Chen, L.; He, L.; Ma, Y.; Zhang, Y., Correlation between bone mineral density and serum trace element contents of elderly males in Beijing urban area. International journal of clinical and experimental medicine 2015, 8 (10), $19250-7$.

22. Sánchez-González, C.; López-Chaves, C.; Gómez-Aracena, J.; Galindo, P.; Aranda, P.; Llopis, J., Association of plasma manganese levels with chronic renal failure. Journal of trace elements in medicine and biology: organ of the Society for Minerals and Trace Elements (GMS) 2015, 31, 78-84. 
23. Vezér, T.; Kurunczi, A.; Náray, M.; Papp, A.; Nagymajtényi, L., Behavioral effects of subchronic inorganic manganese exposure in rats. American journal of industrial medicine 2007, 50 (11), 841-52.

24. Crossgrove, J.; Zheng, W., Manganese toxicity upon overexposure. NMR in biomedicine 2004, 17(8), 544-53.

25. O'Neal, S. L.; Zheng, W., Manganese Toxicity Upon Overexposure: a Decade in Review. Current environmental health reports 2015, 2 (3), $315-28$.

26. Inoue, N.; Makita, Y. J. T. o. M., Neurological aspects in human exposures to manganese. 1996.

27. Li, L.; Yang, X., The Essential Element Manganese, Oxidative Stress, and Metabolic Diseases: Links and Interactions. Oxidative medicine and cellular longevity 2018, 2018, 7580707.

28. Wang, S.; Deng, Z.; Ma, Y.; Jin, J.; Qi, F.; Li, S.; Liu, C.; Lyu, F. J.; Zheng, Q., The Role of Autophagy and Mitophagy in Bone Metabolic Disorders. International journal of biological sciences 2020, 16 (14), 2675-2691.

29. Gao, J.; Feng, Z.; Wang, X.; Zeng, M.; Liu, J.; Han, S.; Xu, J.; Chen, L.; Cao, K.; Long, J.; Li, Z.; Shen, W.; Liu, J., SIRT3/SOD2 maintains osteoblast differentiation and bone formation by regulating mitochondrial stress. Cell death and differentiation 2018, 25 (2), $229-240$.

30. Malecki, E. A., Manganese toxicity is associated with mitochondrial dysfunction and DNA fragmentation in rat primary striatal neurons. Brain research bulletin 2001, 55 (2), 225-8.

31. Rao, K. V.; Norenberg, M. D., Manganese induces the mitochondrial permeability transition in cultured astrocytes. The Journal of biological chemistry 2004, 279 (31), 32333-8.

32. Sriram, K.; Lin, G. X.; Jefferson, A. M.; Roberts, J. R.; Wirth, O.; Hayashi, Y.; Krajnak, K. M.; Soukup, J. M.; Ghio, A. J.; Reynolds, S. H.; Castranova, V.; Munson, A. E.; Antonini, J. M., Mitochondrial dysfunction and loss of Parkinson's disease-linked proteins contribute to neurotoxicity of manganese-containing welding fumes. FASEB journal: official publication of the Federation of American Societies for Experimental Biology 2010, 24 (12), 4989-5002.

33. Goldman; SJ; Taylor; Zhang; Jin; -, S. J., Autophagy and the degradation of mitochondria. 2010.

34. Bocca, B.; Madeddu, R.; Asara, Y.; Tolu, P.; Marchal, J. A.; Forte, G., Assessment of reference ranges for blood Cu, Mn, Se and Zn in a selected Italian population. Journal of trace elements in medicine and biology: organ of the Society for Minerals and Trace Elements (GMS) 2011, 25 (1), $19-26$.

35. Clark, N. A.; Teschke, K.; Rideout, K.; Copes, R., Trace element levels in adults from the west coast of Canada and associations with age, gender, diet, activities, and levels of other trace elements. Chemosphere 2007, 70 (1), 155-64.

36. Lee, J. W.; Lee, C. K.; Moon, C. S.; Choi, I. J.; Lee, K. J.; Yi, S. M.; Jang, B. K.; Yoon, B. J.; Kim, D. S.; Peak, D.; Sul, D.; Oh, E.; Im, H.; Kang, H. S.; Kim, J.; Lee, J. T.; Kim, K.; Park, K. L.; Ahn, R.; Park, S. H.; Kim, S. C.; Park, C. H.; Lee, J. H., Korea National Survey for Environmental Pollutants in the Human Body 2008: heavy metals in the blood or urine of the Korean population. International journal of hygiene and environmental health 2012, 215 (4), $449-57$.

37. Oulhote, Y.; Mergler, D.; Bouchard, M. F., Sex- and age-differences in blood manganese levels in the U.S. general population: national health and nutrition examination survey 2011-2012. Environmental health: a global access science source 2014, 13, 87.

38. Zhang, L. L.; Lu, L.; Pan, Y. J.; Ding, C. G.; Xu, D. Y.; Huang, C. F.; Pan, X. F.; Zheng, W., Baseline blood levels of manganese, lead, cadmium, copper, and zinc in residents of Beijing suburb. Environmental research 2015, 140, 10-7.

39. Rondanelli, M.; Faliva, M. A.; Peroni, G.; Infantino, V.; Tartara, A. J. N. P. C., Essentiality of Manganese for Bone Health: An Overview and Update. 2021,16 (5), $1934578 \times 2110166$.

40. Price; Charles, T. J. O. O. J., Essential Nutrients for Bone Health and a Review of their Availability in the Average North American Diet. 2012.

41. Guilarte, T. R.; Chen, M. K., Manganese inhibits NMDA receptor channel function: implications to psychiatric and cognitive effects. Neurotoxicology 2007, $28(6), 1147-52$.

42. Bouchard, M. F.; Sauvé, S.; Barbeau, B.; Legrand, M.; Brodeur, M.; Bouffard, T.; Limoges, E.; Bellinger, D. C.; Mergler, D., Intellectual impairment in school-age children exposed to manganese from drinking water. Environmental health perspectives 2011, 119 (1), 138-43.

43. Institute of Medicine Panel on, M., In Dietary Reference Intakes for Vitamin A, Vitamin K, Arsenic, Boron, Chromium, Copper, lodine, Iron, Manganese, Molybdenum, Nickel, Silicon, Vanadium, and Zinc, National Academies Press (US) Copyright 2001 by the National Academy of Sciences. All rights reserved.: Washington (DC), 2001.

44. European Food Safety Authority. Tolerable Upper Intake Levels for Vitamins and Minerals. Scientific Committee on Food; Scientific Panel on Dietetic Products, Nutrition and Allergies; 2006.

45. Journal, N. J. E., Scientific opinion on dietary reference values for manganese. 2013, 11 (11).

\section{Figures}




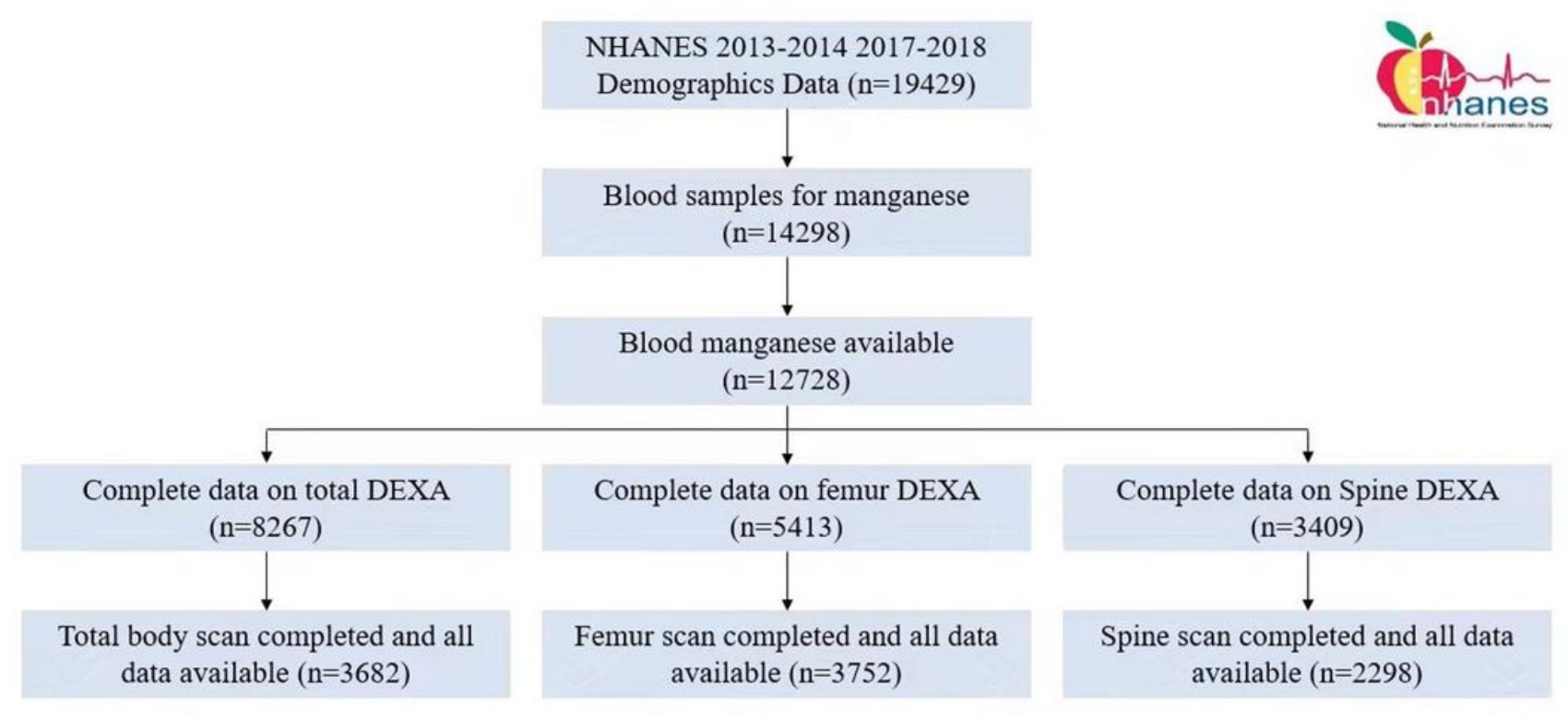

Figure 1

Flow chart. NHANES, National Health and Nutrition Examination Survey; DEXA, Dual Energy X-ray Absorptiometry

\section{Supplementary Files}

This is a list of supplementary files associated with this preprint. Click to download.

- Supplementarytable.docx 